Conflict of interest: BW, LL, WKM, CLL, NZ, MS, and TJS are all current employees of Compass Therapeutics LLC and are partners in the LLC. UE, CC, SO, WG, PB, MO, SQH, JL, WFC, and RT are former employees of Compass Therapeutics and are partners in the LLC. UE, SQH, and WG are now employees of Akrevia Therapeutics. CC is now an employee of CRISPR Therapeutics. $\mathrm{SO}$ is now an employee of Bluebird Bio. RT and JL are now employees of TCR2 Therapeutics. PB is now an employee of Sanofi. MO is now an employee of Bristol-Myers Squibb. WFC is now an employee of Astellas Pharmaceuticals. ACA is a member of the scientific advisory board for Tizona Therapeutics, Compass Therapeutics, and Zumutor Biologics and is a paid consultant for Aximmune. ACA, CL, and $C W$ received research funding from Compass Therapeutics. PB, MS, $J \mathrm{~L}, \mathrm{RT}, \mathrm{CLL}$, and UE are inventors on the following issued U.S. patents held by applicant Compass Therapeutics: patent nos. 10,279,038B2; 10,279,039B2; and 10,279,040B1; these patents cover pharmaceutical compositions comprising CTX-471 and methods of using CTX-471 for treating cancer or inducing antitumor immune response in cancer patients.

Copyright: () 2020, American Society for Clinical Investigation.

Submitted: September 20, 2019

Accepted: January 30, 2020

Published: March 12, 2020.

Reference information: /CI Insight. 2020;5(5):e133647.

https://doi.org/10.1172/jici.

insight.133647.

\section{Differentiated agonistic antibody targeting CD137 eradicates large tumors without hepatotoxicity}

\author{
Ugur Eskiocak, ${ }^{1}$ Wilson Guzman, ${ }^{1}$ Benjamin Wolf, ${ }^{1}$ Christine Cummings, ${ }^{1}$ Lauren Milling, ${ }^{1,2}$ \\ Hsin-Jung Wu, ${ }^{1}$ Michael Ophir, ${ }^{1}$ Conner Lambden, ${ }^{3}$ Pearl Bakhru, ${ }^{1}$ Dana C. Gilmore, ${ }^{1}$ \\ Samantha Ottinger,' Lucy Liu,' William K. McConaughy, ${ }^{1}$ Sunny Q. He, ${ }^{1}$ Chao Wang, ${ }^{3}$ \\ Cheuk Lun Leung, ${ }^{1}$ Jason Lajoie, ${ }^{1}$ William F. Carson IV, ${ }^{1}$ Nora Zizlsperger, ${ }^{1}$ Michael M. Schmidt, ${ }^{1}$ \\ Ana C. Anderson, ${ }^{3}$ Piotr Bobrowicz, ${ }^{1}$ Thomas J. Schuetz, ${ }^{1}$ and Robert Tighe ${ }^{1}$ \\ 'Compass Therapeutics, Cambridge, Massachusetts, USA. ${ }^{2}$ Koch Institute for Integrative Cancer Research, Massachusetts \\ Institute of Technology (MIT), Cambridge, Massachusetts, USA. ${ }^{3}$ Evergrande Center for Immunologic Diseases and \\ Ann Romney Center for Neurologic Diseases, Harvard Medical School and Brigham and Women's Hospital, Boston, \\ Massachusetts, USA.
}

CD137 (4-1BB) is a member of the TNFR superfamily that represents a promising target for cancer immunotherapy. Recent insights into the function of TNFR agonist antibodies implicate epitope, affinity, and IgG subclass as critical features, and these observations help explain the limited activity and toxicity seen with clinically tested CD137 agonists. Here, we describe the preclinical characterization of CTX-471, a fully human IgC4 agonist of CD137 that engages a unique epitope that is shared by human, cynomolgus monkey, and mouse and is associated with a differentiated pharmacology and toxicology profile. In vitro, CTX-471 increased IFN- $\gamma$ production by human T cells in an Fc $\gamma$ receptor-dependent (Fc $\gamma \mathrm{R}$-dependent) manner, displaying an intermediate level of activity between 2 clinical-stage anti-CD137 antibodies. In mice, CTX-471 exhibited curative monotherapy activity in various syngeneic tumor models and showed a unique ability to cure mice of very large $\left(\sim 500 \mathrm{~mm}^{3}\right)$ tumors compared with validated antibodies against checkpoints and TNFR superfamily members. Extremely high doses of CTX-471 were well tolerated, with no signs of hepatic toxicity. Collectively, these data demonstrate that CTX-471 is a unique CD137 agonist that displays an excellent safety profile and an unprecedented level of monotherapy efficacy against very large tumors.

\section{Introduction}

While checkpoint inhibitors targeting PD-1 or CTLA-4 have been transformative therapies in immuno-oncology, they continue to have limited efficacy in the majority of patients in most indications. Agonistic antibodies against costimulatory immune receptors, including members of the TNF receptor superfamily (TNFRSF), have potential to complement checkpoint blockers by directly activating immune cells (1) However, early attempts to develop these antibodies clinically have struggled to find an appropriate balance between efficacy and toxicity.

Cell surface glycoprotein CD137 (also known as 4-1BB and TNFRSF9) is a member of the TNFRSF that is expressed on activated T cells, Tregs, NK cells, monocytes, DCs, and tumor endothelial cells (2). Upon interaction with its cognate ligand, CD137L, CD137 forms stable homotrimers that recruit the TRAF$1 / 2$ signaling adaptors to stimulate downstream activation of the NF- $\mathrm{KB}$ transcriptional pathway. Activation of $\mathrm{CD} 137$ delivers potent costimulatory signals to $\mathrm{CD} 8^{+}$cytotoxic $\mathrm{T}$ cells, promoting cell proliferation, facilitating differentiation into memory cells, and delivering important survival signals. The incorporation of the intracellular signaling domain of CD137 has improved the clinical activity of the second generation of CAR T cell therapies, indicating an important role of CD137 signaling in effective antitumor immunity (3, 4). CD137 stimulation also enhances NK cell proliferation and IFN- $\gamma$ production, and it increases the ability of NK cells to perform antibody-dependent cell-mediated cytotoxicity (ADCC) against tumor cells, demonstrating potential of CD137 agonists to invoke and bridge innate and adaptive immunity (2). 
Multiple preclinical studies demonstrate that antibody agonists of CD137 are highly effective in treating both solid tumors and hematological malignancies in mice (5-8). In addition to monotherapy efficacy, CD137 agonist antibodies can enhance the antitumor effects of tumor-targeted antibodies (9-11), checkpoint inhibitors (12), and other TNFRSF agonists (13), supporting broad clinical combination potential.

Promising preclinical results led to the development and clinical testing of 2 agonistic anti-CD137 antibodies in human cancer patients. Urelumab (BMS-663513), a fully human IgG4 monoclonal antibody developed by Bristol-Myers Squibb, induced inflammatory hepatotoxicity at doses $\geq 0.3 \mathrm{mg} / \mathrm{kg}$, limiting its therapeutic window (14). In contrast, utomilumab (PF-05082566), a fully human IgG2 antibody developed by Pfizer, was safe at doses up to $10 \mathrm{mg} / \mathrm{kg}$ but demonstrated limited clinical efficacy (15). The starkly differing activity profiles of these 2 antibodies are likely related to one or more of the known differences between them: targeted epitope, CD137 ligand blocking capacity, IgG subclass, and the level of intrinsic agonistic activity (16). Neither of these antibodies are mouse cross-reactive, preventing the direct study of their pharmacology and toxicity in mice. However, antibody clone $3 \mathrm{H} 3$ has been widely studied and is known to be a strong agonist of mouse CD137 (17) that induces hepatic inflammation (18-20).

Given the recent insights into the function of TNFR agonist antibodies that implicate epitope, affinity, and IgG subclass $(16,20-24)$ as critical features, an opportunity exists for CD137 agonists to achieve differentiated therapeutic activity and improved safety profile. Here, we describe preclinical characterization of CTX-471, a fully human IgG4 agonist of CD137 that displays a favorable and well-differentiated efficacy-safety profile that is attributed to a unique epitope, optimized affinity, and Fc $\gamma$ receptor-dependent (Fc $\gamma$ R-dependent) activity.

\section{Results}

CTX-471 binds to a unique epitope within CD137 that is conserved in human, cynomolgus monkey, and mouse. CTX-471 is a fully human antibody that binds with moderate monovalent affinity to recombinant human or cynomolgus macaque CD137 $\left(K_{D}=50 \mathrm{nM}\right.$ for human, $61 \mathrm{nM}$ for cyno) and cross-reacts with lower affinity to mouse CD137 $\left(K_{D}=748 \mathrm{nM}\right.$; Supplemental Figure 1A; supplemental material available online with this article; https://doi.org/10.1172/jci.insight.133647DS1). CTX-471 similarly binds to primary human or cyno $\mathrm{T}$ cells with approximately equal affinity $\left(\mathrm{EC}_{50}=1.4 \mathrm{nM}\right.$ for human, 0.66 $\mathrm{nM}$ for cyno) and with lower affinity to murine $\mathrm{T}$ cells $\left(\mathrm{EC}_{50}=36 \mathrm{nM}\right.$; Supplemental Figure $\left.2, \mathrm{~A}-\mathrm{C}\right)$. To obtain the most pharmacologically relevant data from preclinical mouse models, CTX-471 was affinity matured to generate CTX-471-AF, which has increased affinity for both recombinant mouse CD137 $\left(K_{D}\right.$ $=86 \mathrm{nM}$, Supplemental Figure $1 \mathrm{~A})$ and on murine $\mathrm{T}$ cells $\left(\mathrm{EC}_{50}=2.8 \mathrm{nM}\right.$; Supplemental Figure 2C). Both values are similar to binding of parental CTX-471 to human CD137, supporting the use of CTX471-AF as an affinity-matched mouse surrogate.

CTX-471 binds CD137 at a nonligand competitive epitope, as demonstrated by the ability of recombinant CD137L to bind to a preformed complex of CTX-471 and CD137 (Figure 1A). As reported previously, CD137L also binds to a preformed complex of CD137 and urelumab, while utomilumab blocks ligand binding (16). In domain mapping experiments using truncated versions of CD137, CTX-471 binds to the membrane proximal cysteine rich domains 3-4 (CRD3-4), similar to utomilumab and in contrast to urelumab, which binds to CRD1-2 (Figure 1B). CTX-471 binding is significantly reduced by mutations at amino acid K114 with additional contributions from E111, T113, N126, I132, and P135 (Supplemental Figure 1B), consistent with an epitope in CRD3-4 on a face of CD137 directed away from the ligand binding site (Figure 1C). In contrast, urelumab binds at a membrane distal epitope centered on amino acid R41 (16). Point mutation K113A in mouse CD137 (analogous to K114 in human) eliminates binding of CTX-471$\mathrm{AF}$, confirming that the epitope is conserved between species (Figure 1, D and E). Mutation of amino acid Y40 in mouse CD137 (analogous to R41 in human CD137) disrupts binding of the murine CD137-specific surrogate antibody $3 \mathrm{H} 3$ (Figure 1D), suggesting that it binds to CRD1 at a highly similar epitope to where urelumab engages the human receptor (Figure 1E).

$C T X-471$ stimulates primary $T$ cells from human, monkey, and mouse with intermediate potency. The activity of TNFR agonist antibodies is influenced by Fc $\gamma \mathrm{R}$ interactions that promote receptor clustering (20-24). We selected human IgG4 as the backbone for CTX-471 based on the ability of this isotype to engage the $\mathrm{F}_{\mathrm{c}}$ receptors $\mathrm{F}_{\mathrm{c}} \gamma \mathrm{RI}$ (CD64) and $\mathrm{F}_{\mathrm{c}} \gamma \mathrm{RIIb}(\mathrm{CD} 32 \mathrm{~b})$ to drive receptor cross-linking, while avoiding binding to Fc $\gamma$ RIIIa (CD16a) and ADCC-mediated depletion of immune effector cells expressing CD137 $(25,26)$. 
A

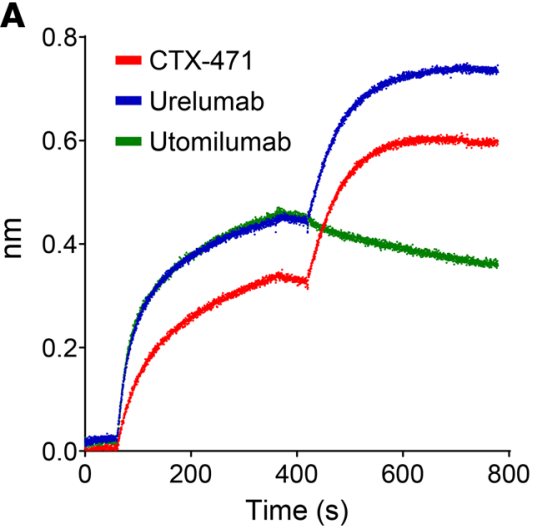

B
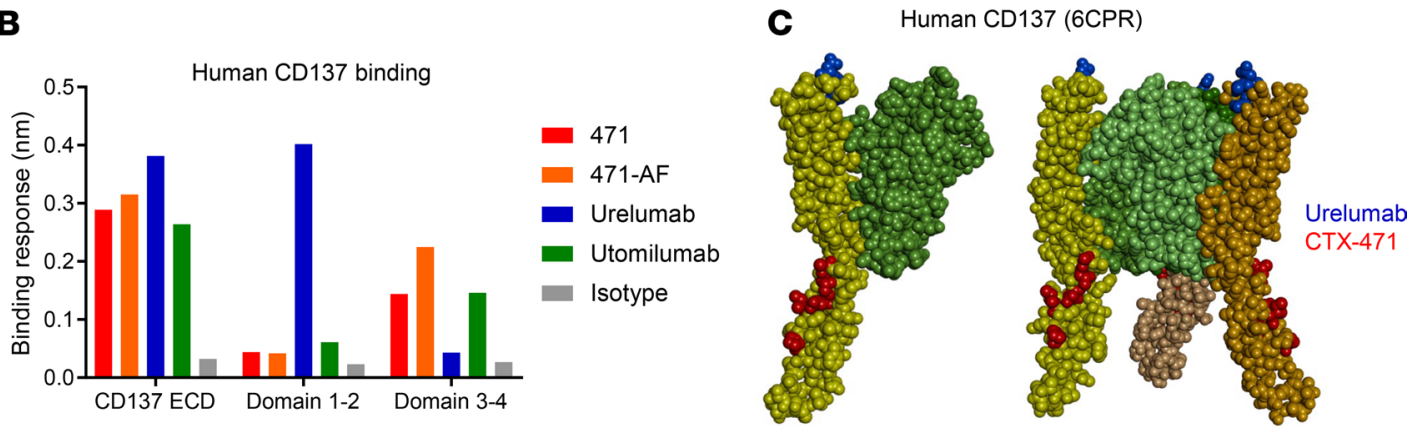

D

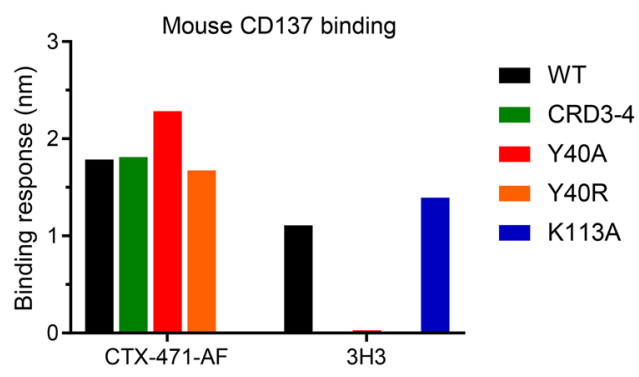

E

Mouse CD137 (6MKZ)

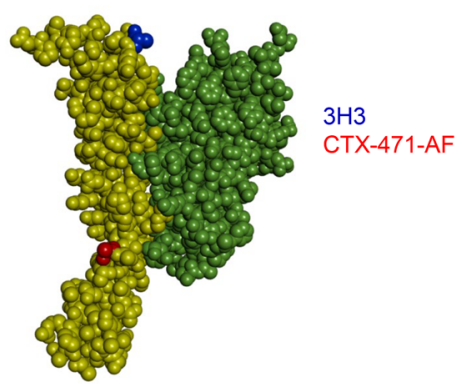

Figure 1. CTX-471 binds to a unique epitope on CD137. (A) Binding traces from Bio-Layer Interferometry (BLI) experiments testing ability of recombinant CD137L to bind to preformed complexes of CD137 and stated antibody. (B) Max BLI response measured for binding of full-length or truncated forms of human CD137 to tested antibodies. (C) Mapping of identified contact residues for binding of CTX-471 (red) or urelumab (blue) onto crystal structure of human CD137/CD137L complex (PDB 6CPR) based on mutational analyses. (D and E) BLI measurements for binding of mouse CD137 truncations and point mutations to tested antibodies with mapping of identified contact residues onto crystal structure of the mouse CD137/CD137L complex (PDB 6MKZ).

In coculture experiments with anti-CD3 activated primary $\mathrm{T}$ cells and $\mathrm{CHO}$ cells engineered to express CD32b, stimulation with CTX-471 or CTX-471-AF induced IFN- $\gamma$ production from human T cells in a dose-dependent manner, with a low nanomolar $\mathrm{EC}_{50}$ (Figure 2A). Importantly, CTX-471 showed an intermediate level of activation that fell between the superagonist activity of urelumab and the very weak activity of utomilumab (Figure 2A). CTX-471 consistently increased activation of $\mathrm{T}$ cells from multiple human donors (Figure 2B). When WT CHO cells lacking expression of $\mathrm{CD} 32 \mathrm{~b}$ were used in the coculture assay, there was no appreciable IFN- $\gamma$ induction, demonstrating the Fc $\gamma$ R-dependent nature of the agonistic activity of CTX-471 (Figure 2C). CTX-471 and CTX-471-AF also induced IFN- $\gamma$ production from mouse (Supplemental Figure 2D) and cynomolgus monkey (Supplemental Figure 2E) T cells in analogous coculture assays, confirming cross-functionality across species. The $3 \mathrm{H} 3$ antibody displayed superagonist activity in the mouse assay, further supporting its use as a functional surrogate for urelumab (Supplemental Figure 2D).

CD137L potentiates $C T X-471-i n d u c e d ~ N F-\kappa B$ signaling. Signaling through receptors of the TNFR superfamily, such as $\mathrm{CD} 137$, requires receptor trimerization and clustering for efficient signaling (27). To measure the ability of CD137 antibodies to induce clustering, we built a split-luciferase complementation system in 
A

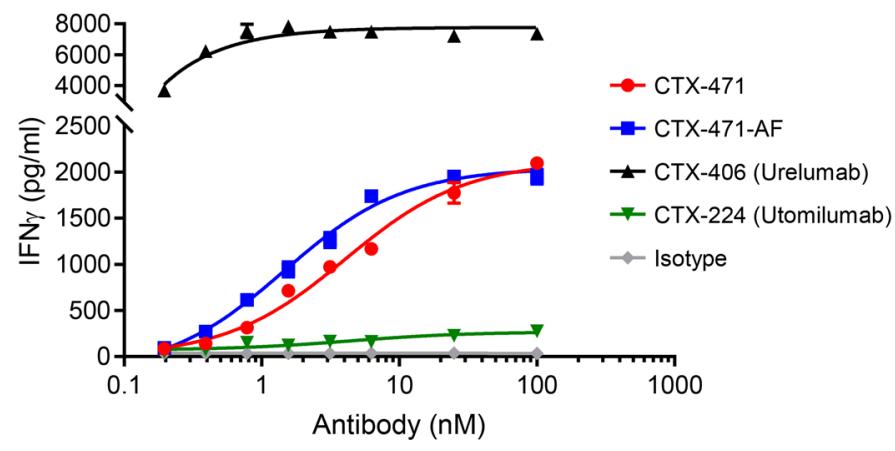

C

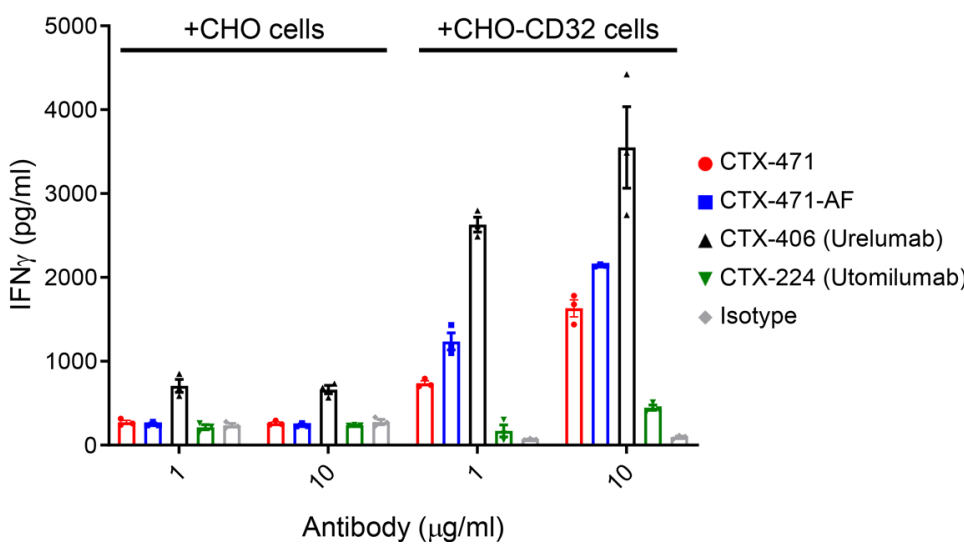

B

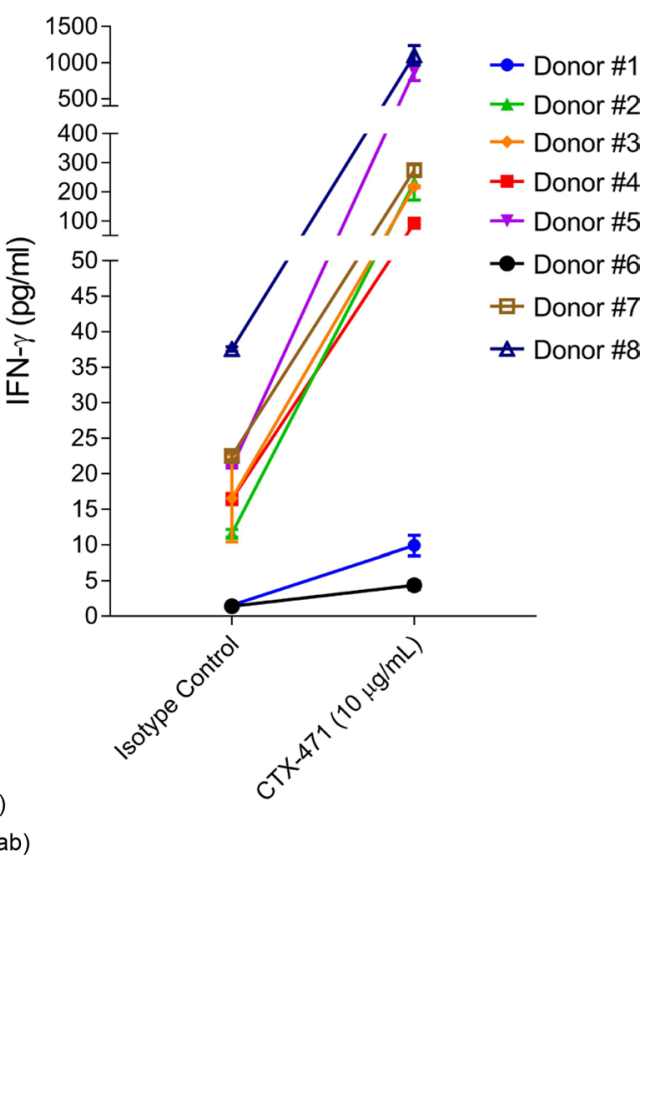

Figure 2. CTX-471 costimulates primary T cells with Fc $\gamma$ R-dependent activity. (A-C) IFN- $\gamma$ secretion after a 3-day coculture of primary T cells and CHO cells engineered to express Fc $\gamma$ RIlb (CD32b). Freshly isolated human T cells were stimulated with soluble anti-CD3 (clone OKT3) and anti-CD137 antibodies in the presence of $\mathrm{CHO}-\mathrm{CD} 32 \mathrm{~b}$ ( $\mathbf{A}$ and $\mathbf{B}$ ) or $\mathrm{CHO}$ (C) cells. Representative data from $n=3-4$ experiments $(\mathbf{A}$ and $\mathbf{C})$. All data presented as mean $\pm \mathrm{SEM}$.

which clustering of CD137 induced by cross-linked antibodies causes the $\mathrm{N}$ - and C-termini of firefly luciferase to colocalize, interact, and refold into a functional enzyme. A molecular analogue of urelumab induced extensive, dose-dependent clustering, with single-digit nanomolar doses causing a 12-fold increase in signal over the isotype control (Figure 3A). In comparison, CTX-471 induced moderate clustering of CD137, with a signal increase ranging from 3 - to 11 -fold (Figure $3 \mathrm{~A}$ ).

As CD137 signaling events culminate in NF-kB activation (28), we measured the ability of CTX-471 to elicit signaling in NF-kB-luciferase reporter cells and determined whether signaling was affected by CD137's natural ligand, CD137L. In the absence of the ligand, CTX-471, urelumab, and utomilumab induced NF- $\mathrm{kB}$ signaling in a dose-dependent manner when cross-linked (Figure 3, B-D). In the presence of suboptimal concentrations of CD137L, the amount of NF-kB signaling induced by CTX-471 was enhanced, while activity of urelumab was largely unaffected and signaling from utomilumab was substantially decreased (Figure 3, B-D). The attenuated activity of utomilumab in the presence of CD137L is consistent with the observation that this antibody competes with ligand binding (Figure 1A). CD137L binding similarly increased IFN- $\gamma$ production from T cells treated with CTX-471 (Figure 3E), while it had no effect on cells treated with urelumab or utomilumab (data not shown).

CTX-471-AF monotherapy achieves high rates of complete tumor regression in multiple syngeneic mouse models. The mouse cross-reactivity and cross-functionality of CTX-471-AF and CTX-471 allowed the testing of these antibodies in syngeneic mouse tumor models in which the host animals have fully intact immune systems. In the CT-26 murine colon carcinoma model, both CTX-471-AF and CTX-471 demonstrated potent antitumor activity across a broad dose-range in mice bearing established $\left(50-75 \mathrm{~mm}^{3}\right)$ tumors (Figure $4 \mathrm{~A}$ and Supplemental Figure 3A), resulting in a high rate of complete cures and significantly improved overall survival (Figure 4B and Supplemental Figure 3B). At its most efficacious dose level of $150 \mu \mathrm{g}$, CTX-471-AF 
A

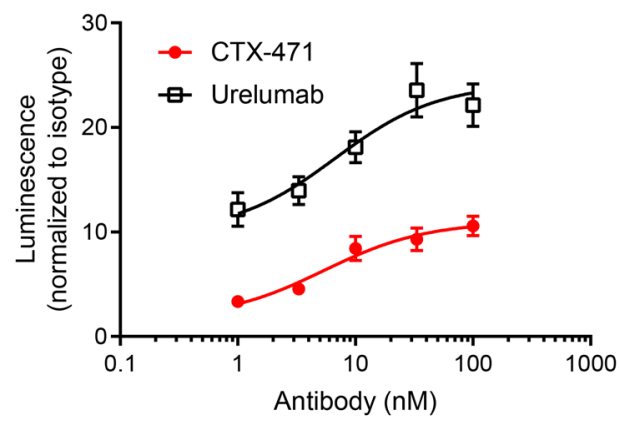

C

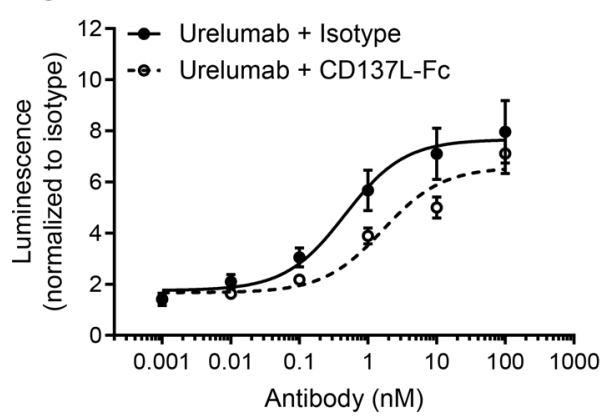

E
B

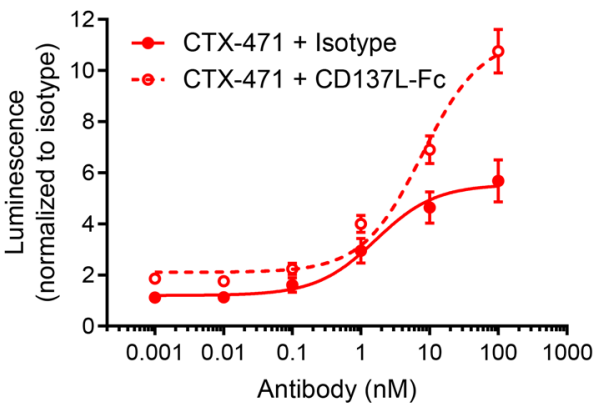

D

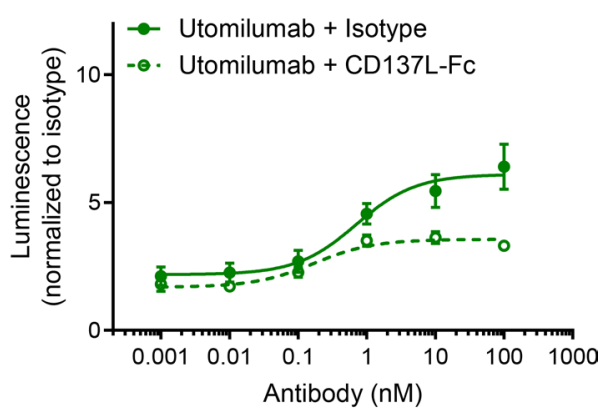

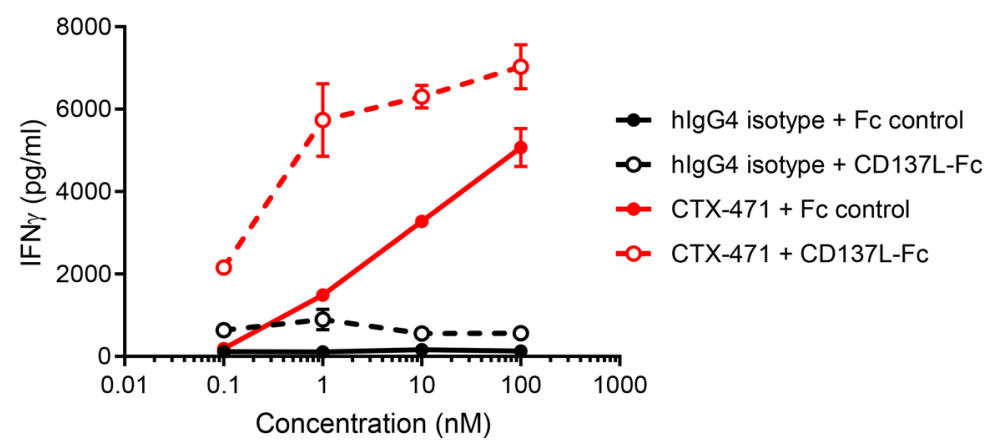

Figure 3. CTX-471-induced activity is further enhanced by CD137 ligand. (A) HEK-SplitCD137 cells were incubated with cross-linked anti-CD137 antibodies for 4 hours. The cells were then lysed and the level of CD137 cross-linking was determined by measuring luminescence of firefly luciferase. (B-D) HEK-293T cells expressing CD137 and a minimal NF-KB reporter were cocultured in the presence of plate-bound isotype or CD137L-Fc, along with cross-linked CTX-471 (B), urelumab (C), or utomilumab (D) for 4 hours. The cells were then lysed, and the level of $\mathrm{NF}-\mathrm{KB}$ signaling was determined by measuring the luminescence of firefly luciferase. All data are the average of $n=3$ independent experiments, presented as mean \pm SEM. (E) IFN- $\gamma$ secretion after a 3 -day coculture of anti-CD3 activated primary $\mathrm{T}$ cells with CHO-CD32b cells and CTX-471 with or without CD137L-Fc.

achieved complete tumor regressions in $100 \%$ of treated mice ( 8 of 8 mice) (Figure $4 \mathrm{~A}$ ). Curative efficacy was also observed at the lowest tested dose level of $12.5 \mu \mathrm{g} /$ mouse with both antibodies. Mice that experienced complete responses were rechallenged with a second inoculation of CT-26 tumor cells on the opposing flank 71-88 days after the last treatment. Complete protection from tumor regrowth was observed, indicating the establishment of long-term antitumor immunity (Figure 4B and Supplemental Figure 3B). CTX-471-AF treatment also achieved complete cures across a wide dose range in the A20 B cell lymphoma and the orthotopic EMT6 triple-negative breast cancer models, leading to significantly improved overall survival and longterm protection from tumor rechallenge (Figure 4, C and D, and Supplemental Figure 3C).

In order to understand the effect of epitope and in vitro potency on in vivo activity, we compared CTX-471-AF with the urelumab-like mouse surrogate antibody 3H3. At the higher dose levels, both CTX471-AF and $3 \mathrm{H} 3$ achieved complete tumor regressions and significant extension of overall survival (Supplemental Figure 4A). At the lowest tested dose level of $12.5 \mu \mathrm{g} /$ mouse, CTX-471-AF achieved complete 
A
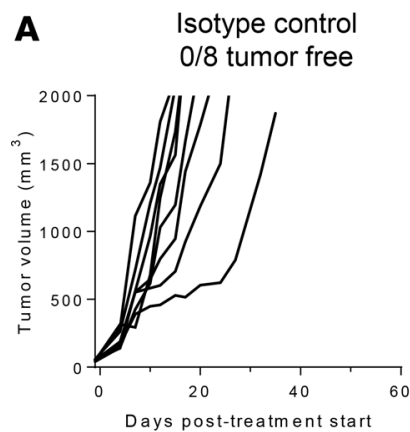

CTX-471-AF $(150 \mu \mathrm{g})$ $8 / 8$ tumor free

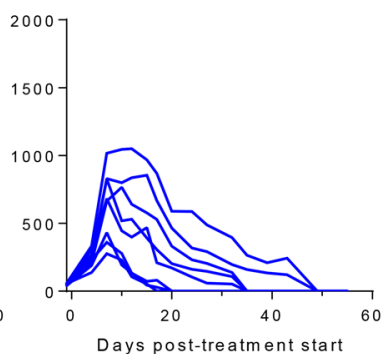

CTX-471-AF $(50 \mu \mathrm{g})$ $4 / 8$ tumor free

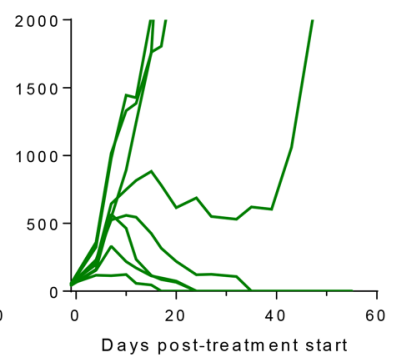

CTX-471-AF $(12.5 \mu \mathrm{g})$

$6 / 8$ tumor free

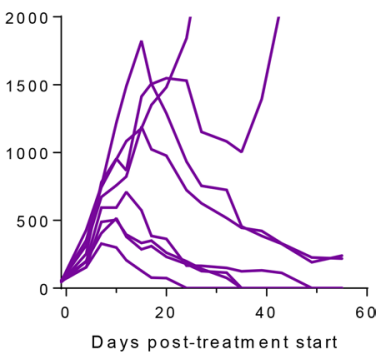

B
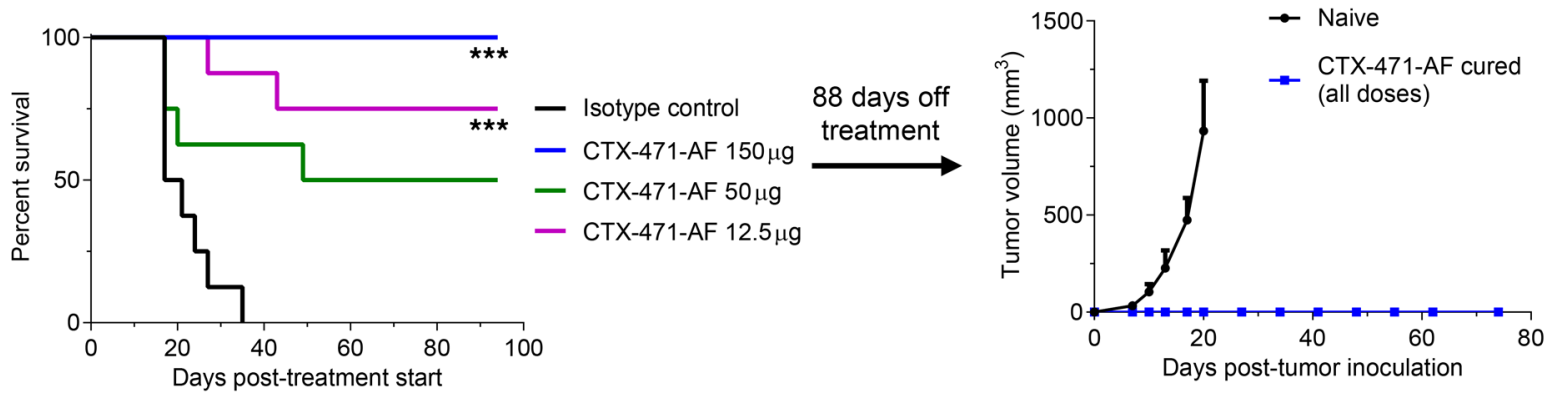

C

Isotype control 2/8 tumor free
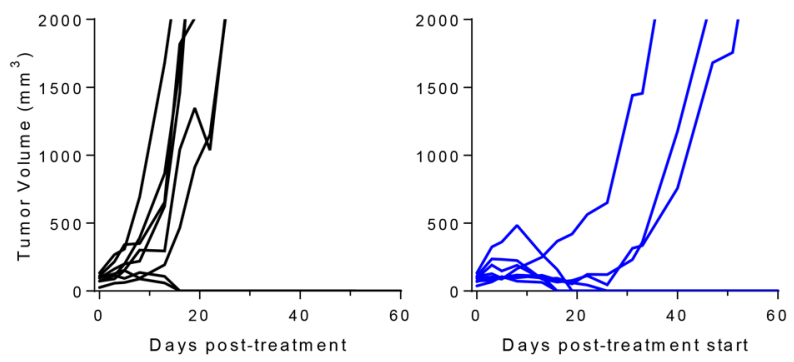

CTX-471-AF $(50 \mu \mathrm{g})$ $2 / 8$ tumor free

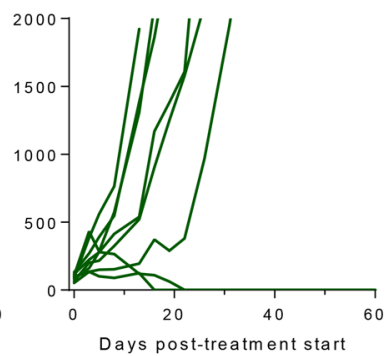

CTX-471-AF $(12.5 \mu \mathrm{g})$ $4 / 8$ tumor free

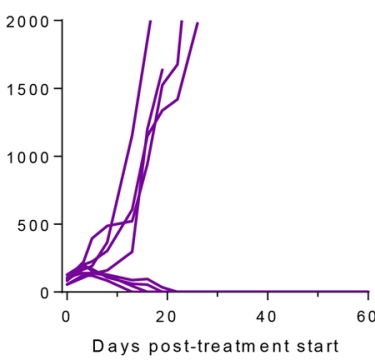

D
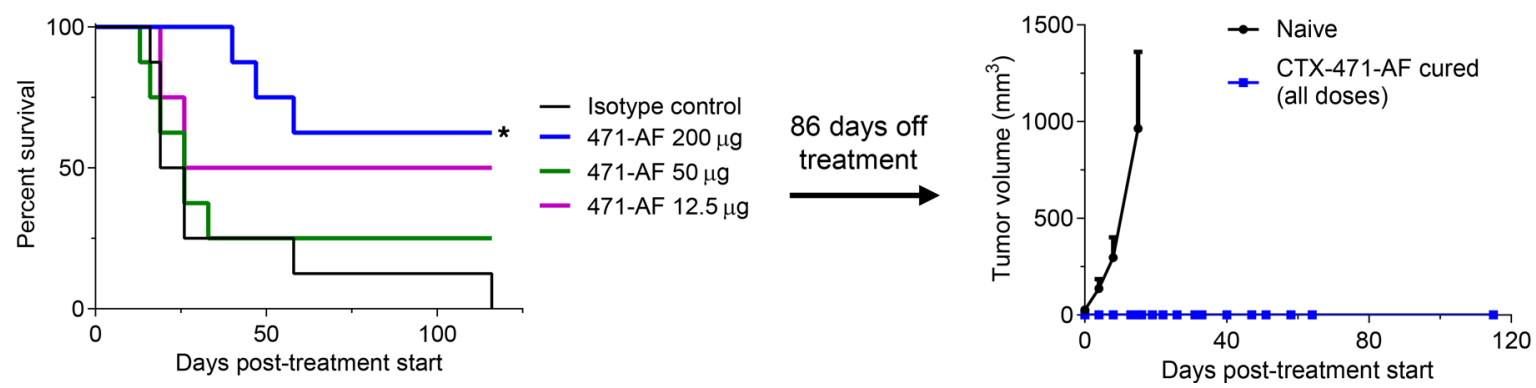

Figure 4. CTX-471-AF achieves high rates of complete tumor regression in mouse models. (A) Tumor growth rates of individual mice with established (50-75 mm3) CT-26 tumors following i.p. administration of CTX-471-AF on days 0, 3, 6, and 9. (B) Kaplan-Meier survival curves of mice in A and growth curves from tumor rechallenge of cured mice 88 days after last treatment. (C) Tumor growth rates of individual mice with established (75-100 mm ${ }^{3}$ ) A20 tumors following i.p. administration of CTX-471-AF on days 0, 7, 14, 21 and 28. (D) Kaplan-Meier survival curves of mice in C and growth curves from tumor rechallenge of cured mice 86 days after last treatment. Log-rank test followed by Bonferroni's multiple comparison analysis was performed to determine the statistical significance of overall survival with CTX-471-AF versus control $\left({ }^{*} P<0.05\right.$, $\left.{ }^{* * *} P<0.001\right)$. 
tumor regressions in 7 of 8 mice $(88 \%)$, leading to a significant benefit in overall survival $(P=0.0003)$ compared with control treated mice (Supplemental Figure 4A). In contrast, 3H3 led to tumor regression in 3 of 8 mice (43\%) and did not significantly improve overall survival (Supplemental Figure 4A), suggesting that CTX-471-AF was more efficacious in vivo, despite significantly lower in vitro potency. The antitumor activity elicited by $3 \mathrm{H} 3$ was accompanied by significant splenomegaly, along with increased plasma levels of the proinflammatory cytokines TNF- $\alpha$, IFN- $\gamma$, and IL-27 (Supplemental Figure 4, B-E). CTX-471-AF did not induce significant changes in these parameters at any dose level, suggesting that CTX-471-AF causes a weaker systemic activation of the immune system than $3 \mathrm{H} 3$ (Supplemental Figure 4, B-E).

$C T X-471-A F$ selectively and profoundly reprograms the tumor microenvironment. Across a wide dose range, treatment with CTX-471-AF induced a profound influx of CD45+ leukocytes within the majority of CT-26 tumors when compared with tumors from isotype control-treated animals (Figure 5A). While the overall proportions of intratumoral $\mathrm{CD}^{+}$and $\mathrm{CD}^{+} \mathrm{T}$ cells did not change significantly in response to CTX-471$\mathrm{AF}$ (Figure 5, B and C), the observed expansion of the $\mathrm{CD} 45^{+}$compartment indicates an increase in the total number of tumor-infiltrating $\mathrm{T}$ cells. There was also an expansion of antigen-positive $\mathrm{T}$ cells in the tumor, as measured by AH-1 pentamer staining (Supplemental Figure 5A). Conversely, the level of immunosuppressive Tregs within tumors decreased significantly at all tested doses of CTX-471-AF, as compared with isotype control treatment (Figure 5D). Among tumor infiltrating leukocytes, CD137 is most highly expressed on Tregs (23), which may render these cells uniquely susceptible to depletion by CTX-471-AF through antibody-dependent cell-mediated phagocytosis (ADCP).

Treatment with CTX-471-AF was associated with significantly decreased coexpression of the immunoinhibitory exhaustion markers TIGIT and PD-1 on the surface of intratumoral CD8 ${ }^{+}$and CD $4^{+} \mathrm{T}$ cells, indicating that CTX-471-AF therapy either protected tumor-infiltrating $\mathrm{T}$ cells from exhaustion or reversed the exhausted phenotype (Figure 5, E and F). Tumor-infiltrating lymphocytes (TILs) in CTX-471-treated mice also demonstrated increased metabolic uptake and mitochondrial activity as reported previously (29, 30) (Supplemental Figure 5B). The proportion of $\mathrm{CD} 11 \mathrm{~b}^{+} \mathrm{F} 4 / 80^{+}$macrophages was reduced in the tumors of mice treated with CTX-471-AF (Figure 5G). The macrophages remaining within the tumor microenvironment following treatment displayed a repolarization to an antitumor M1 phenotype, as evidenced by higher expression of the M1 markers CD38 and iNOS (Figure 5H). NK cells in the tumor shifted to a more mature or cytotoxic phenotype with increased expression of CD11b (Supplemental Figure 5C).

Distinct from the robust immunophenotypic changes observed within tumors following CTX-471AF treatment, the effects on peripheral immunophenotypes were much milder. CTX-471-AF did not cause any major change in the numbers or proportions of effector $\mathrm{T}$ cells, Tregs, or macrophages in the spleen (Supplemental Figure 6, A-D, and H). Counter to what was observed in tumors, the frequency of TIGIT and PD-1 coexpression on $\mathrm{CD}^{+}$and $\mathrm{CD}^{+} \mathrm{T}$ cells was increased within spleens, along with an increased frequency of $\mathrm{CD}^{+} \mathrm{T}$ cells possessing a CD44 $4^{+} \mathrm{CD} 62 \mathrm{~L}^{-}$effector memory phenotype (Supplemental Figure 6, E-G).

In contrast to CTX-471-AF, treatment with $3 \mathrm{H} 3$ resulted in substantial changes in the peripheral immune system, including significant expansion of liver $\mathrm{CD}^{+} \mathrm{T}$ cells at all tested dose levels (Supplemental Figure 7, A-D). 3H3 also dramatically increased the frequency of $\mathrm{PD}^{+} \mathrm{TIGIT}^{+} \mathrm{T}$ cells in the spleens and livers of treated mice, compared with more mild changes in the CTX-471-AF-treated animals (Supplemental Figure 7, E-H). Together, these data suggest that the stronger agonistic signaling from $3 \mathrm{H} 3$ may be associated with broader $\mathrm{T}$ cell activation and exhaustion compared with the more moderate signaling from CTX-471-AF.

CTX-471-AF promotes acquisition of memory precursor and effector gene programs in CD8 TILs. To further delineate how CTX-471-AF and $3 \mathrm{H} 3$ affect $\mathrm{CD} 8^{+}$TILs, we isolated CD8 ${ }^{+}$TILs from CT-26 tumor-bearing mice treated with either CTX-471-AF, 3H3, or isotype control and performed droplet-based scRNA sequencing (scRNA-Seq). After data filtering, we obtained expression data from 8696 cells. Graph-based clustering revealed 11 cell clusters across CTX-471-AF, 3H3, and isotype treatment with nonuniform distributions of the 3 conditions across clusters (Figure 5I and Supplemental Figure 8A). Gene expression across the 3 largest clusters 0,1 , and 2 demonstrated differential enrichment between control-, CTX-471-AF-, or 3H3-treated mice (Figure 5J and Supplemental Tables 1-4).

Consistent with better elicitation of effector T cell functions after $3 \mathrm{H} 3$ and CTX-471-AF treatment, we observed elevated enrichment of pathways such as $\mathrm{T}$ cell activation in clusters 0 and 2 compared with cluster 1 (Supplemental Figure 8B). We further quantified the expression of a memory-precursor signature (31), 


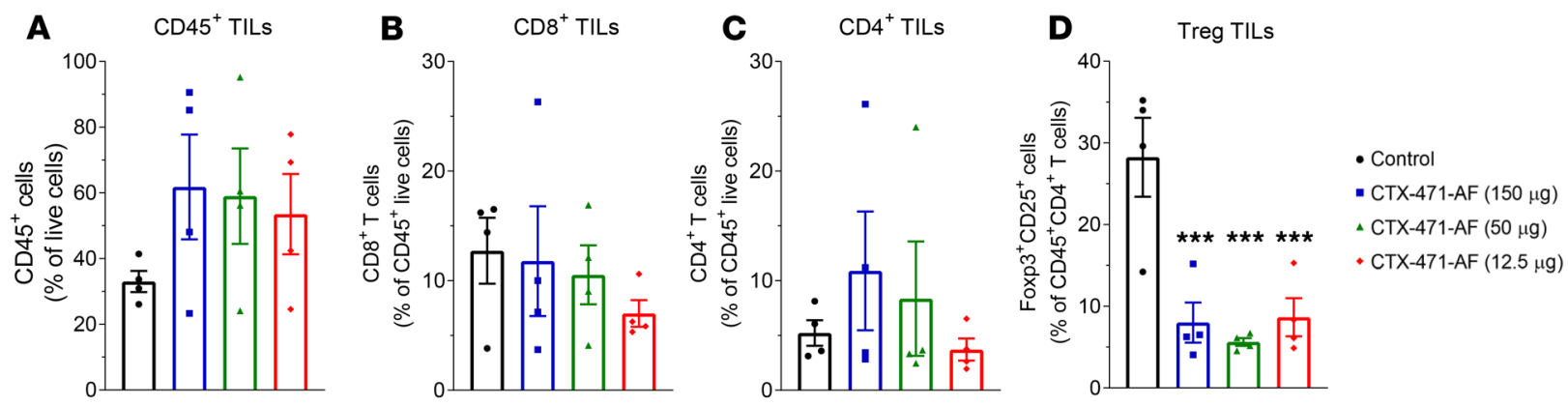

E $\quad \mathrm{TIGIT}^{+} \mathrm{PD}-1^{+} \mathrm{CD} 8^{+} \mathrm{TILS}$

F $\quad \mathrm{TIGIT}^{+} \mathrm{PD}-1^{+} \mathrm{CD} 4^{+} \mathrm{TILS}$

G

TAMs
H M1 polarized TAMs
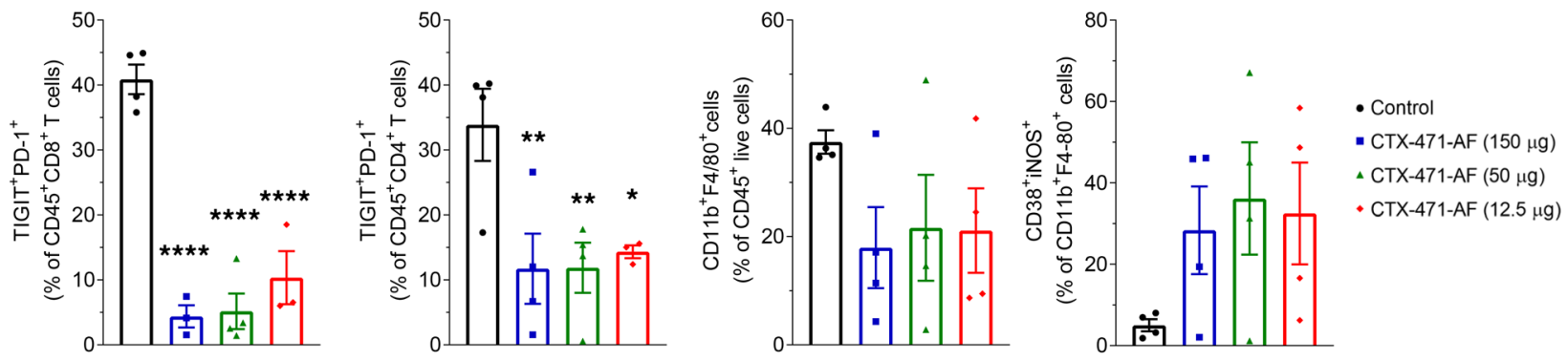

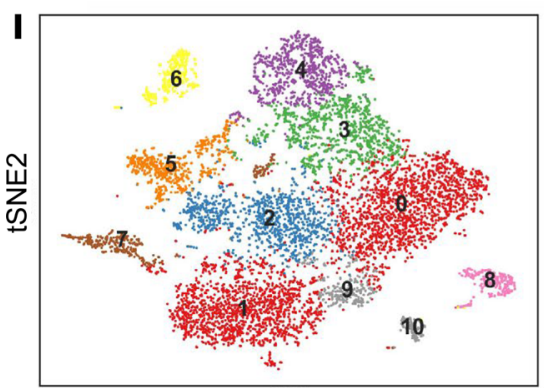

tSNE1

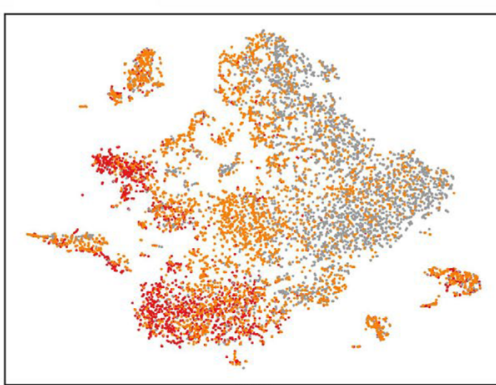

tSNE1
$\mathbf{K}$

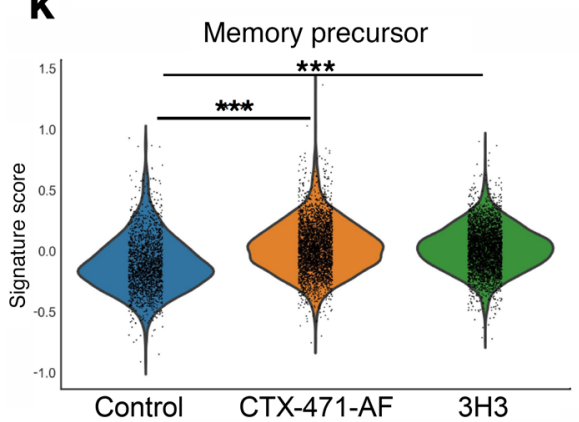

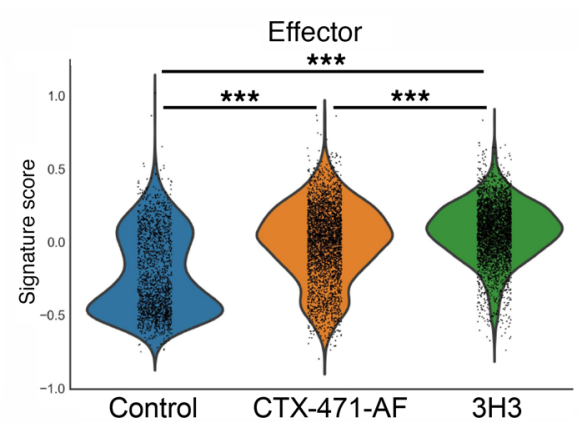

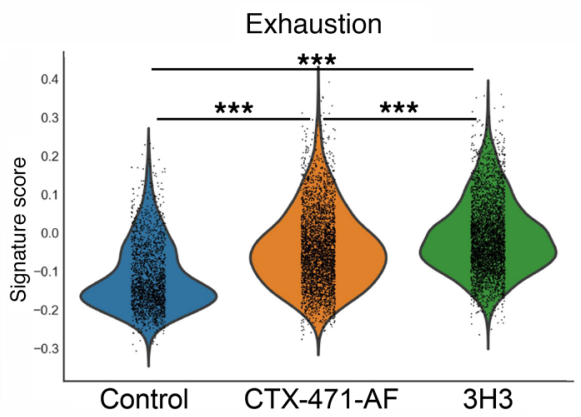

Figure 5. CTX-471 profoundly reprograms the tumor immune environment. (A-H) Mice with established CT-26 tumors ( $n=4$ per group) were treated with CTX-471-AF on days $0,3,6$, and 9 . Tissues were harvested on day 11, and dissociated tumors were analyzed by flow cytometry. CD45 tumor infiltrating leukocytes (TILs; A); fraction of CD8 ${ }^{+}$T cells (B), CD4+ $T$ cells (C), and Tregs (D) within TILs; TIGIT+PD-1+ double-positive cells within CD8 ${ }^{+}$T cells (E), and CD4+ T cells (F); tumor associated macrophages (TAM; G) and M1 polarized TAMs (H) are shown. Statistical significance was determined using 1-way ANOVA followed by Bonferroni's multiple comparison compared with control treatment groups $\left({ }^{*} P<0.05,{ }^{* *} P<0.01,{ }^{* * *} P<0.001,{ }^{* * * *} P<0.0001\right)$. All data are presented as mean \pm SEM. (I) t-SNE plot showing unsupervised clustering of the SCRNA profiles of CD8 ${ }^{+}$TILs from CT-26 tumor-bearing mice treated with isotype, CTX-471-AF, or 3H3. t-SNE plot highlighting cells from isotype- (red), CTX-471-AF- (orange), and 3H3-treated (gray) mice. (J) Heatmap of differentially expressed genes between clusters $(0,1$, and 2 ) that show differential enrichment in cells from isotype-, CTX-471-AF-, and 3H3-treated mice. (K) Violin plots showing expression of a memory-precursor $C D 8^{+} T$ cell signature (left), effector $C D 8^{+} \mathrm{T}$ cell signature (middle), and a dysfunction/exhaustion signature (right) in the scRNA profiles of CD8+ TILs from isotype- (blue), CTX-471-AF- (orange), and 3H3-treated (green) mice. ${ }^{* *} P<0.001$ by Benjamini-Hochberg corrected Tukey's multiple comparisons test. 
A

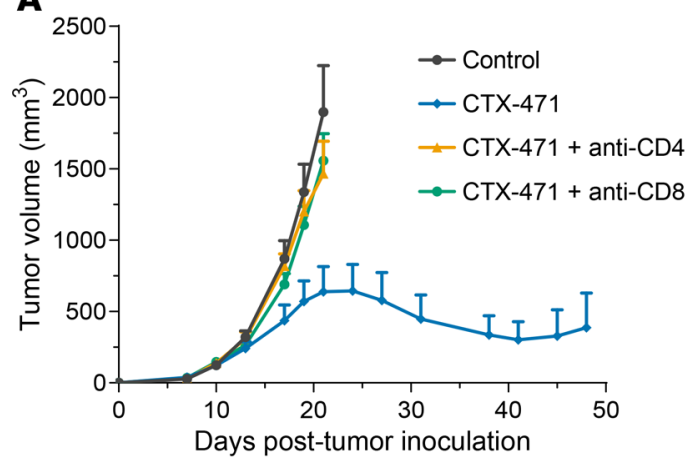

B

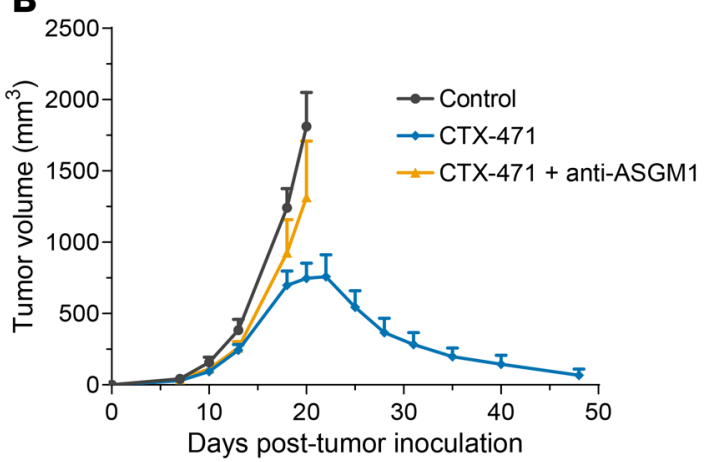

C

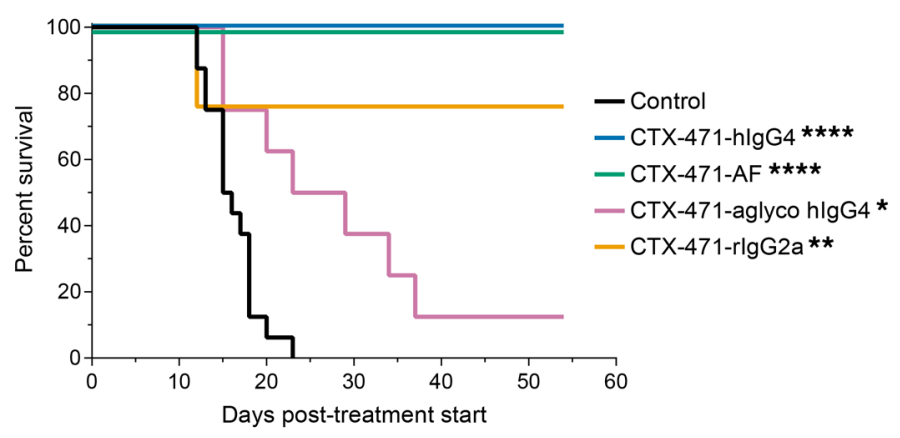

D

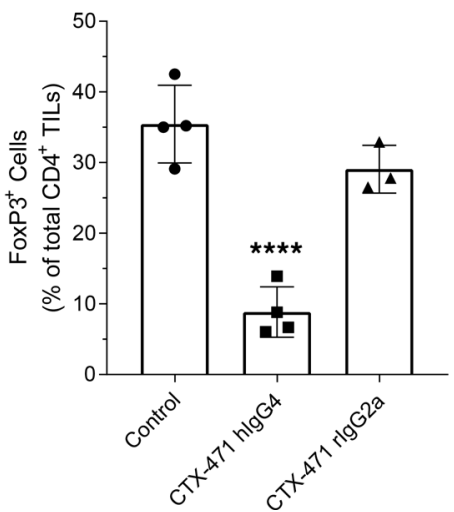

Figure 6. CTX-471 efficacy requires T and NK cells, as well as FcyR engagement. (A) Effect of CTX-471 on tumor growth in the absence of T cells. Mice ( $n=10$ per group) were depleted of T cells by administering $500 \mu \mathrm{g} /$ mouse of CD4 (GK1.5) or CD8 (YTS 169.4) antibodies on days $-1,0,5,10,15$, and 20 after CT-26 tumor cell implantation. Mice received $150 \mu \mathrm{g} /$ mouse CTX-471 on days 6, 9, 12, 19, and 26. (B) Effect of CTX-471 on tumor growth in the absence of NK cells. Mice ( $n=8$ per group) were depleted of NK cells by administering $50 \mu \mathrm{L} / \mathrm{mouse}$ of asialo-GM1 antibody (anti-ASGM1) on days $-1,0,5,10,15$, and 20 after CT-26 tumor cell implantation. Mice received $150 \mu \mathrm{g} /$ mouse CTX-471 on days 7, 10, 13, 20, and 27. (C) Effect of CTX471 on overall survival in the absence of Fc $\gamma R$ engagement. Mice with established CT-26 tumors were treated with 150 $\mu$ g/mouse of CTX-471-AF or CTX-471 with human IgG4, aglycosylated human IgG4, or rat IgG2a isotypes on days $0,3,6$, and 9 . (D) Flow cytometric analysis of CT-26 tumor infiltrating Tregs on day 9 following administration of CTX-471 with hlgG4 or rlgG2a isotype on days 0 , 3, and 6. Statistical significance was determined using Log-rank test (C) or 1-way ANOVA (D) followed by Bonferroni's multiple comparison test compared with control treatment groups $\left({ }^{*} P<0.05\right.$, $\left.{ }^{* *} P<0.01,{ }^{* * * *} P<0.0001\right)$. All data presented as mean $\pm \mathrm{SEM}$.

an effector T cell signature (31), and an exhaustion/dysfunction signature (32) in the CD8 $8^{+}$TILs across the 3 groups (Figure $5 \mathrm{~K}$ ). We saw that the $\mathrm{CD} 8^{+}$TILs from both CTX-471-AF- and 3H3-treated mice showed more memory-precursor/stem-like cells relative to the $\mathrm{CD} 8^{+} \mathrm{TILs}$ from isotype-treated controls (Figure $5 \mathrm{~K})$. Furthermore, although the CD8 $8^{+}$TILs from both CTX-471-AF- and 3H3-treated mice showed acquisition of effector and exhaustion gene programs, significant differences in the proportion of cells expressing these programs indicated that CTX-471-AF treatment does not push cells as hard toward exhaustion/dysfunction as $3 \mathrm{H} 3$ treatment; instead, it sustains an intermediate level of effector function.

The antitumor activity of $C T X-471$ is driven by $F C \gamma R$ engagement and requires the coordinated involvement of both $T$ cells and NK cells. To identify the relative contribution of specific immune effector cell types to the antitumor activity of CTX-471, we treated CT-26 tumor-bearing mice with CTX-471 in the context of immune subset depletion. Antibody-based depletion of either $\mathrm{CD} 8^{+} \mathrm{T}$ cells, $\mathrm{CD} 4^{+} \mathrm{T}$ cells, or NK cells resulted in a nearly complete loss of antitumor activity (Figure 6, A and B). These observations indicate that the tumor rejections induced by CTX-471 therapy require the coordinated activity of both innate and adaptive immune effector cells.

Human IgG4 antibodies bind to mouse Fc receptors $F_{c} \gamma \mathrm{RI}$ and $\mathrm{Fc}_{\mathrm{C}} \mathrm{RIIb}$ through the $\mathrm{Fc}_{\mathrm{c}}$ domain, albeit with lower affinity compared with the corresponding human receptors $(25,33)$, and are able to induce ADCP by mouse macrophages (34). To explore the contribution of Fc $\gamma \mathrm{R}$ binding on antitumor activity, a set of CTX471 variants was engineered with different isotypes varying in Fc receptor engagement and effector function. Consistent with previous findings, treatment with CTX-471 and CTX-471-AF resulted in potent antitumor activity that resulted in complete tumor regressions in all mice and $100 \%$ overall survival (Figure 6C). 

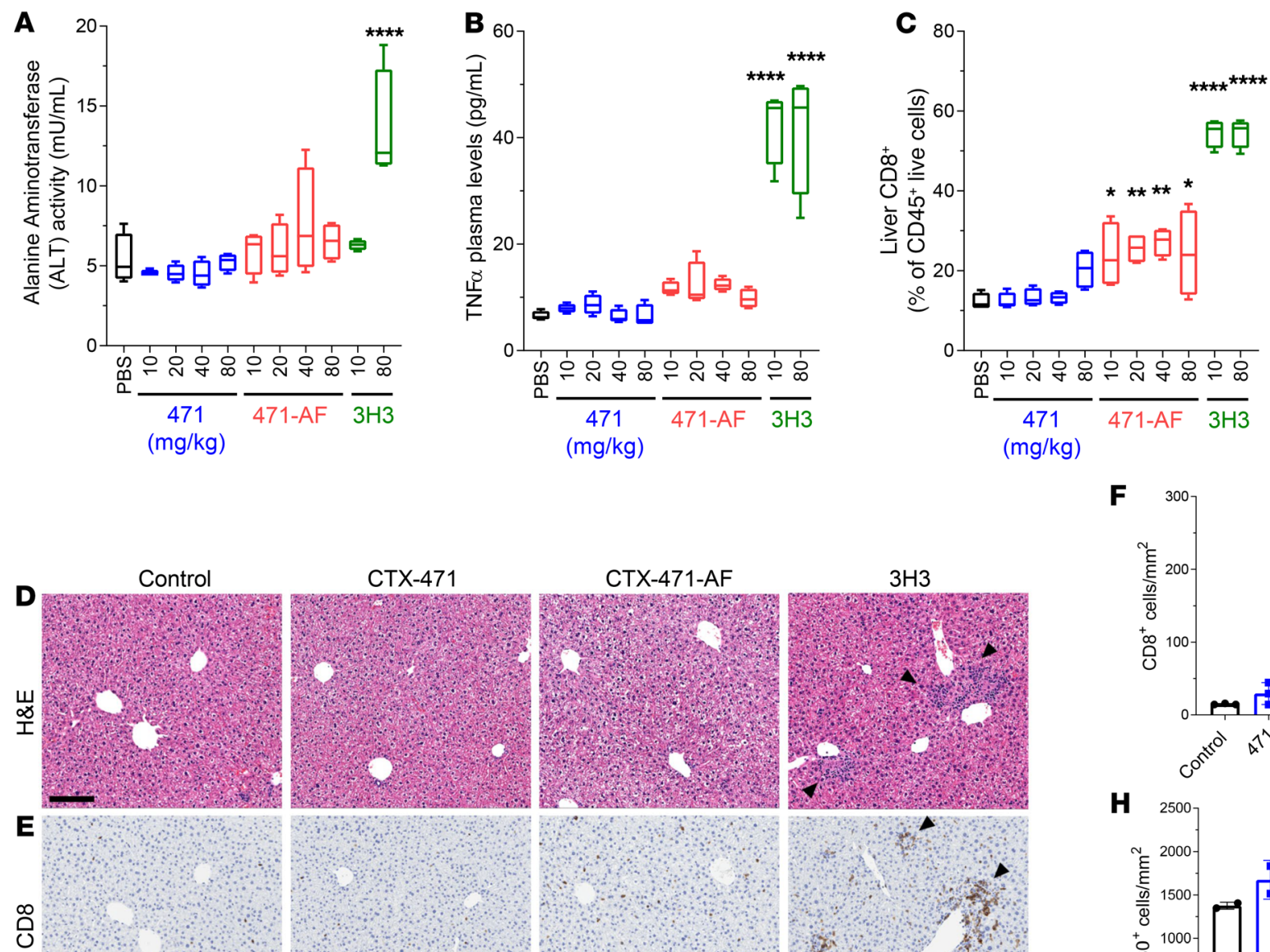

H
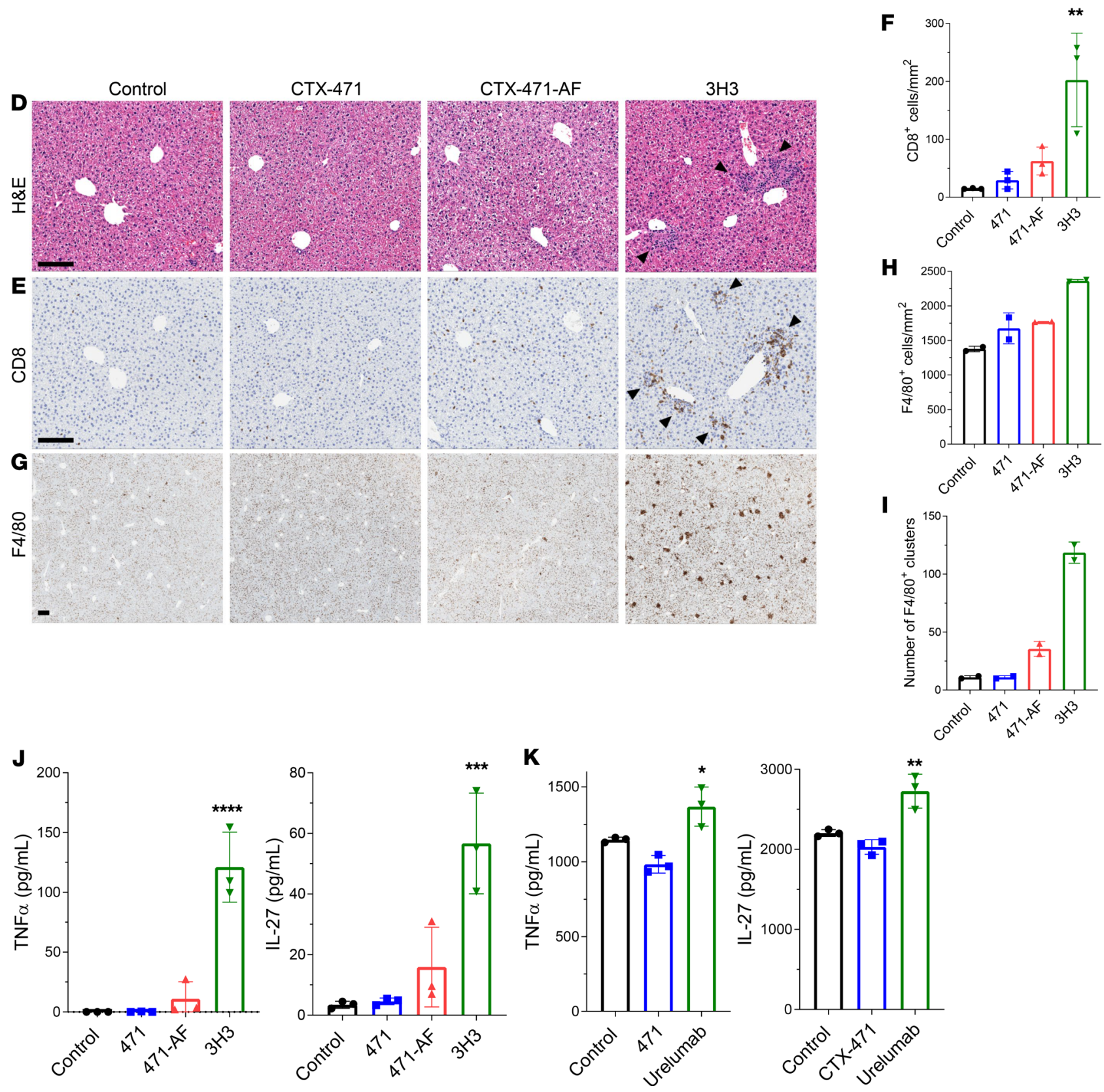
Figure 7. High doses of CTX-471 and CTX-471-AF do not induce hepatic inflammation. (A-I) Nontumor-bearing mice ( $n=4$ in each treatment group) were administered with 10-80 mg/kg CTX-471, CTX-471-AF, or 3H3 i.v. on days $0,7,14$, and 21. Plasma, spleens, and livers were collected on day 28. Alanine aminotransferase (ALT) activity (A) and TNF- $\alpha$ (B) concentrations were determined from plasma. CD8 $8^{+}$cell (C) levels in the livers were determined by flow cytometry. Formalin-fixed paraffin-embedded tissues were stained for H\&E (D) or with antibodies against CD8 (E and F) and F4/80 (G-I). Mean cell densities of CD8 ${ }^{+}$cells $(\mathbf{F})$ and F4/80+ cells (H), and number of F4/80 clusters (I; defined as $>10$ cells), are shown. Scale bars: $200 \mu \mathrm{m}$. Arrowheads indicate lymphocyte clusters. (J) Mouse BM-derived macrophages were stimulated with CpG ODN (10 $\mu \mathrm{g} / \mathrm{mL})$, along with the indicated soluble antibodies ( $50 \mathrm{nM}$ ), for 48 hours, and levels of TNF- $\alpha$ and IL-27 were measured in supernatants. (K) Primary human monocytederived macrophages were stimulated with CpG ODN $(10 \mu \mathrm{g} / \mathrm{mL})$, along with the indicated soluble antibodies (50 $\mathrm{nM})$ for 48 hours, and levels of TNF- $\alpha$ and IL-27 are measured in supernatants. One-way ANOVA with Bonferroni's post hoc test was performed to determine the statistical significance of treatment versus control $\left({ }^{*} P<0.05,{ }^{* *} P<0.01,{ }^{* *} P<0.001,{ }^{* * *} P<0.0001\right)$.

A variant of CTX-471 with point mutation N303A (equivalent to N297A in ref. 35) that prevents Fc glycosylation and $\mathrm{Fc} / \mathrm{Fc} \gamma$ receptor binding showed severely reduced efficacy that led to cures in only 1 of 8 mice (13\%), highlighting the importance of Fc $\gamma$ R-mediated clustering.

Clone $3 \mathrm{H} 3$ is a rat IgG2a antibody; therefore, a series of experiments was performed comparing isotype-matched versions of CTX-471 and $3 \mathrm{H} 3$ to better define the relative effects of binding domain and $\mathrm{FC}$ isotype on their differentiated activity. Rat IgG2a Fc domains bind preferentially to inhibitory Fc receptors in the mouse, mediating Fc clustering but with diminished ability to induce ADCC/ADCP in mice compared with human IgG4 (36). 3H3-hIgG4 behaved similarly to 3H3-rIgG2a in the CT-26 model, leading to significantly higher peripheral activation compared with CTX-471, as measured by systemic proinflammatory cytokines and $\mathrm{T}$ cell infiltration and activation/exhaustion in the liver and spleen (Supplemental Figure 9). CTX-471 also demonstrated better antitumor activity compared with the isotype-matched 3H3-hIgG4, demonstrating that the wider therapeutic window of CTX-471 compared with $3 \mathrm{H} 3$ is primarily a product of its differentiated binding epitope and affinity (Supplemental Figure 9).

In contrast, the human IgG4 isotype was critical to the ability of CTX-471 to reduce Tregs in the tumor, as a rat IgG2a variant of the molecule had no effect (Figure 6D). Similarly, CTX-471-rIgG2a did not reduce the frequency of $\mathrm{PD}^{+} / \mathrm{TIGIT}^{+} \mathrm{T}$ cells in the tumor, as observed for CTX-471-hIgG4 (Supplemental Figure 9). CTX-471-rIgG2a was still highly effective in the CT-26 tumor model, with 6 of 8 cures, but it did not match the 8 of 8 cures of the hIgG4 version (Figure 6C). Collectively, these data suggest that the antitumor activity of CTX-471 critically depends on Fc receptor-mediated clustering, with Fc-mediated depletion of Tregs playing a small but complementary role, and that CTX-471 — as a human IgG4 - represents an optimal combination of binding domain and $\mathrm{Fc}_{\mathrm{c}}$ isotype.

High doses of CTX-471 and CTX-471-AF do not induce hepatic inflammation. The first CD137 antibody to enter the clinic, urelumab, demonstrated dose-dependent moderate-to-severe hepatic toxicity (14). Urelum$\mathrm{ab}$ is not mouse cross-reactive; however, the mouse-specific anti-CD137 agonist antibody 3H3, which binds to a similar epitope as urelumab, has been shown to cause hepatic inflammation in mice (18-20). To assess the potential of CTX-471, CTX-471-AF, and $3 \mathrm{H} 3$ to induce liver inflammation, we administered up to 80 $\mathrm{mg} / \mathrm{kg}$ of these antibodies to nontumor-bearing mice by weekly i.v. injections.

At the end of the 4-week dosing period, $3 \mathrm{H} 3$ treatment induced a mild but significant increase in spleen and liver weights, whereas CTX-471 and CTX-471-AF had no effects (Supplemental Figure 10, A and B). Serum levels of transaminases (e.g., ALT and AST) were increased with 3H3 but not with CTX-471 or CTX-471-AF (Figure 7A and Supplemental Figure 10C). 3H3-induced hepatic inflammation has been shown to involve CD137-mediated activation of liver-specialized macrophages known as Kupffer cells, leading to the production of proinflammatory cytokines including IL-27 and TNF- $\alpha$ that, in concert with autoantigen presentation by the Kupffer cells, triggers a $\mathrm{CD} 8^{+} \mathrm{T}$ cell-driven autoimmune hepatitis (18). Consistently, we observed elevated plasma levels of proinflammatory cytokines TNF- $\alpha$ (Figure 7B), expansion of $\mathrm{CD}^{+} \mathrm{T}$ cells in livers and spleens (Figure 7, C-F, and Supplemental Figure 10D), and expansion and foci formation of Kupffer cells (Figure 7, G-I) in mice treated with 3H3. In contrast, these parameters were either not affected by CTX-471 and CTX-471-AF or the effects were much milder compared with $3 \mathrm{H} 3$ (Figure 7, A-I; and Supplemental Figure 10, A-D).

Treatment of murine BM-derived macrophages with $3 \mathrm{H} 3$ in vitro significantly increased expression of both IL-27 and TNF- $\alpha$, while CTX-471 treatment had no measurable effect and CTX-471-AF induced a mild but nonsignificant increase (Figure 7J). 3H3 treatment also increased production of IL- 6 and IL-1 $\beta$ by CpG-stimulated murine macrophages (Supplemental Figure 10, E and F). To confirm this observation with primary human cells, peripheral blood monocytes from healthy human donors were matured to macrophages 
A
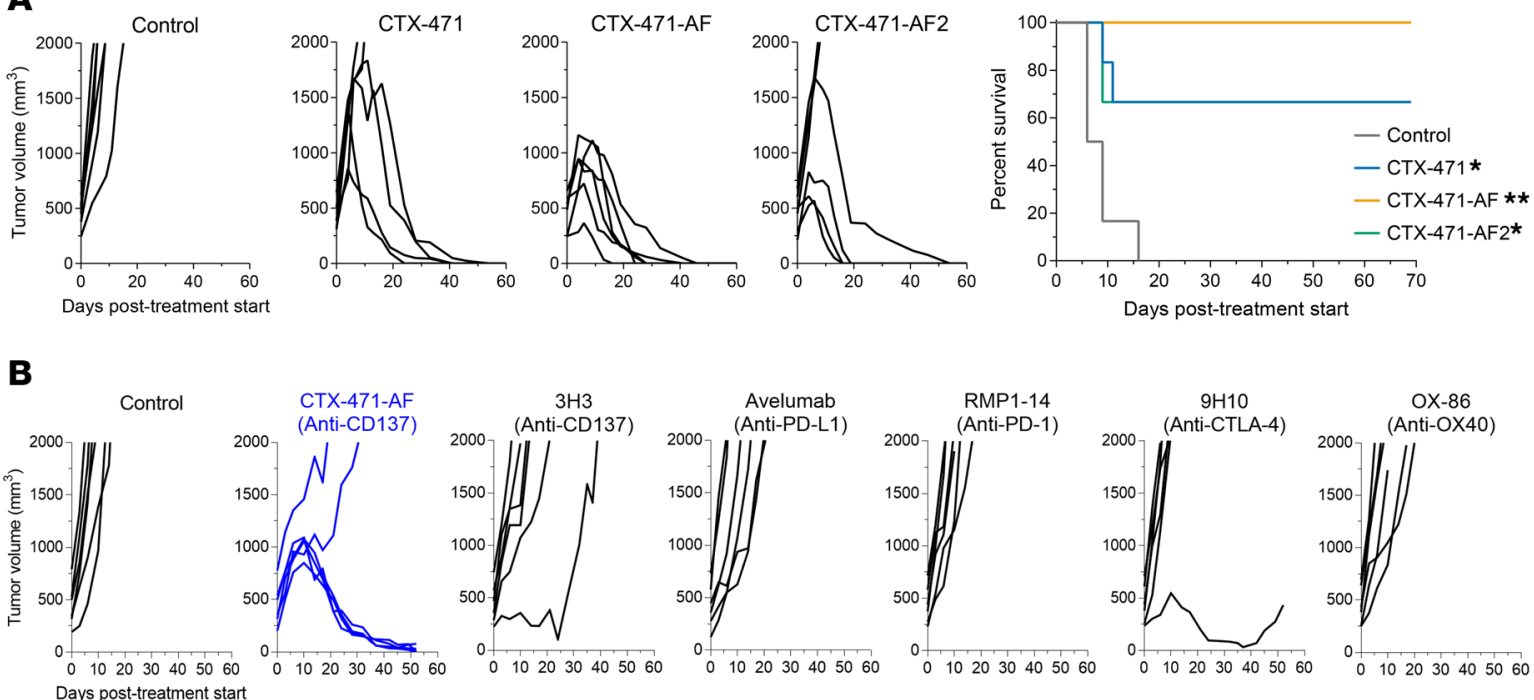

C

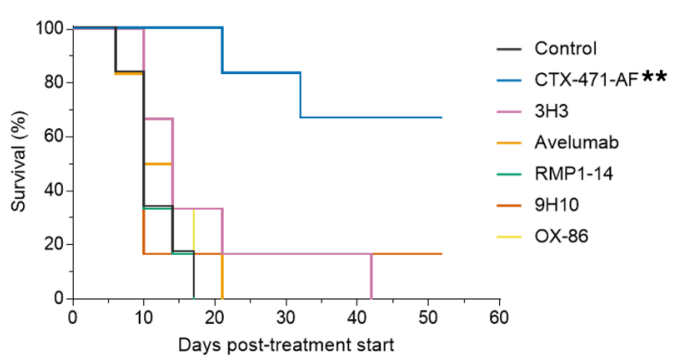

E
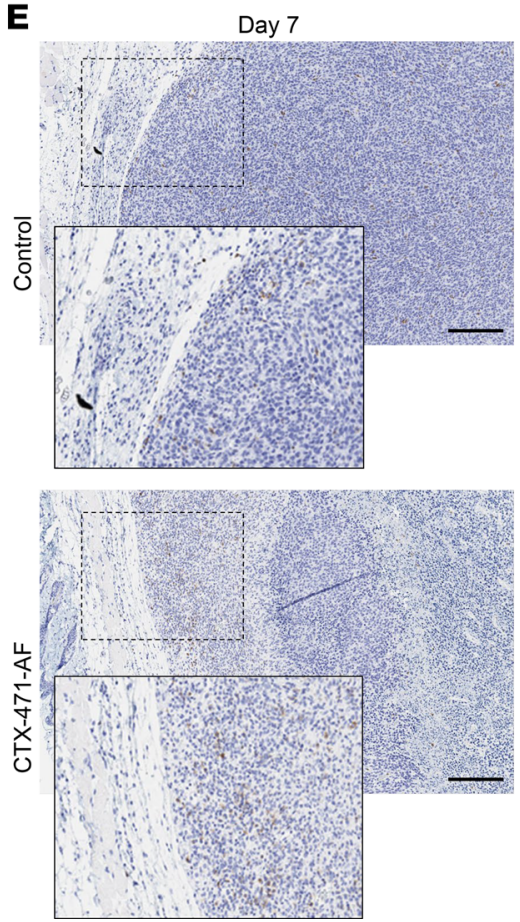

D

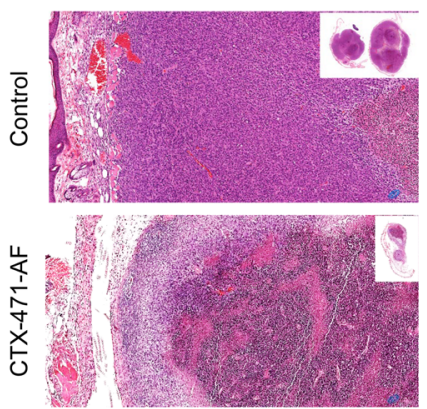

Day 14

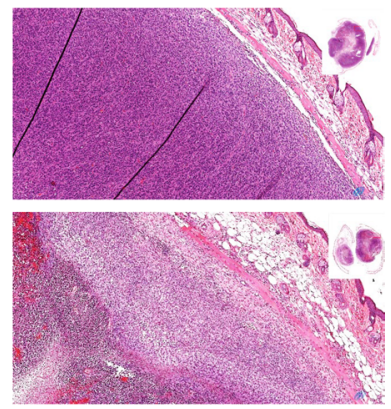

Day 14
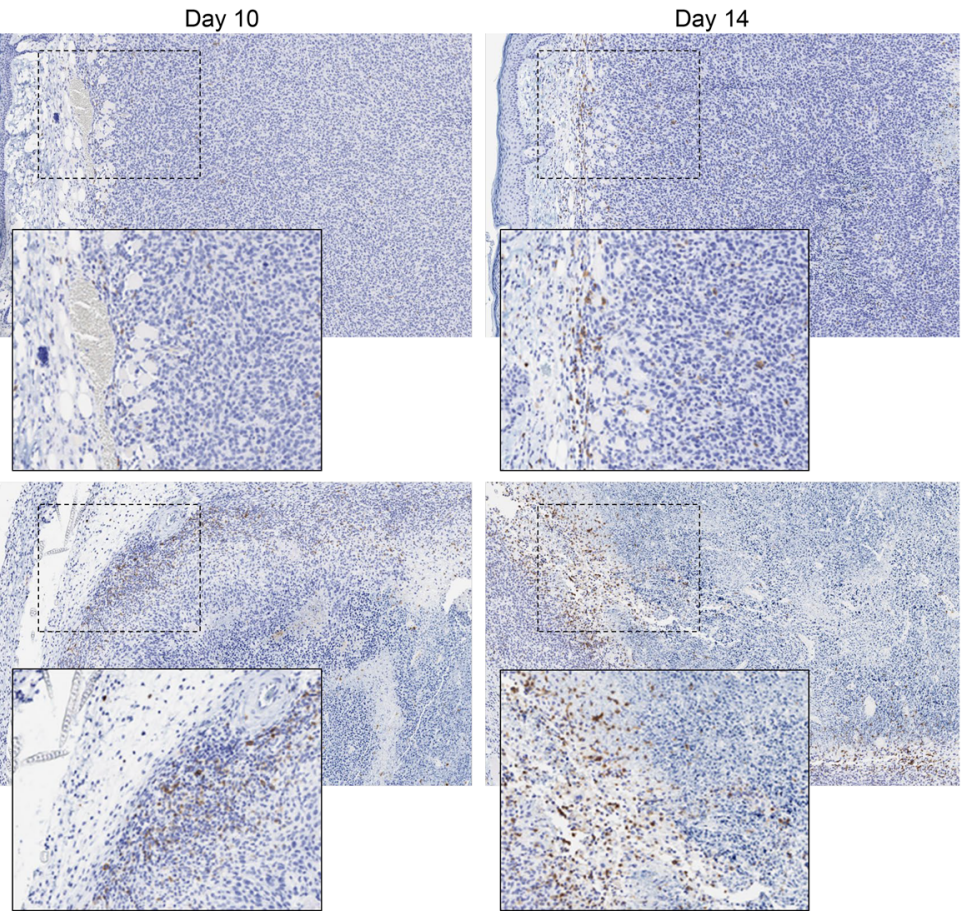

Figure 8. CTX-471-AF eradicates large tumors. (A) Overall survival and tumor growth rates of individual mice with large $\left(\sim 500 \mathrm{~mm}^{3}\right)$ CT-26 tumors following i.p. administration of $150 \mu \mathrm{g}$ /mouse CTX-471, CTX-471-AF, and CTX-471-AF2 on days 0, 3, 6, and 9. (B) Tumor growth rates of individual mice with large $\left(\sim 450 \mathrm{~mm}^{3}\right)$ CT-26 tumors following i.p. administration of CD137 agonists (CTX-471-AF or 3H3; $25 \mu \mathrm{g} / \mathrm{mouse}$ on days 0 , 7, and 14), checkpoint inhibitors (Avelumab, RMP1-14, or 9H10; $200 \mu \mathrm{g} /$ mouse on days 0, 3, and 6), or an OX40 agonist (OX-86; 200 $\mu \mathrm{g} / \mathrm{mouse}$ on days 0, 3, and 6). (C) Kaplan-Meier survival curves of mice in B. Statistical significance was determined using Log-rank test (A and C) followed by Bonferroni's multiple 
comparison compared with control treatment groups ( ${ }^{*} P<0.05$, $\left.{ }^{*} P<0.01\right)$. (D and E) Histological analysis of CT-26 tumors on days 7,10 , and 14 following i.p. administration of $25 \mu \mathrm{g} /$ mouse CTX-471-AF or isotype control on day 0. Formalin-fixed paraffin-embedded tissues were stained for H\&E (D) or with antibodies against CD8 (E). Scale bars: $200 \mu \mathrm{m}$.

in vitro and stimulated in a similar fashion with $\mathrm{CpG}$ and anti-CD137 antibodies. Human macrophages treated with CTX-471 did not show any changes in TNF- $\alpha$ production, whereas macrophages treated with urelumab had increased TNF- $\alpha$ and IL-27 production (Figure 7K and Supplemental Figure 10G).

$C T X-471-A F$ has a unique ability to cure mice of large tumors. Considering the dramatic efficacy observed with CTX-471 and CTX-471-AF in the CT-26 tumor model, we decided to increase the stringency and therapeutic relevance of the model by allowing tumors to grow to $\sim 500 \mathrm{~mm}^{3}$ before starting treatment. We first compared the efficacy of CTX-471 with CTX-471-AF and CTX-471-AF2, a higher affinity variant that binds mouse CD137 with monovalent $K_{D}$ of $10 \mathrm{nM}$. All 3 versions of the antibody showed an ability to completely regress large CT-26 tumors and significantly extend overall survival (Figure 8A). CTX-471-AF performed the best, achieving a 100\% complete regression rate, suggesting that an intermediate affinity for CD137 is optimal for antitumor activity. Consistent with prior studies, all cured mice showed durable protection from tumor rechallenge (Supplemental Figure 11).

Comparison studies were performed against anti-CD137 clone $3 \mathrm{H} 3$ and a panel of well-characterized immuno-oncology antibodies targeting PD-1, PD-L1, CTLA-4, or OX40 that have demonstrated activity against smaller CT-26 tumors in the literature (37-40). In the context of large CT-26 tumors $(\sim 450$ $\mathrm{mm}^{3}$ ), however, these antibodies all failed to induce complete tumor regressions, substantially reduce tumor growth, or enhance overall survival (Figure 8, B and C). In contrast, CTX-471-AF induced complete tumor regression in 4 of 6 mice. Antitumor efficacy of CTX-471-AF was associated with generation of an extensive necrotic core in the tumor surrounded by inflammatory granulation (Figure 8D). Unlike control tumors, where $\mathrm{CD}^{+} \mathrm{T}$ cells were low in number and confined to the periphery, CTX-471-AF treatment induced $\mathrm{CD}^{+} \mathrm{T}$ cell expansion and infiltration (Figure $8 \mathrm{E}$ ).

\section{Discussion}

Immunomodulation via antibody-mediated agonism of TNFR superfamily members is a promising strategy for treating cancer either alone or in combination with checkpoint blockade (1). As a potent and inducible costimulator of both cytotoxic T cells and NK cells, CD137 was among the first TNFR to be identified as a target for cancer immunotherapy (2). The disparate safety and pharmacology profiles of the 2 anti-CD137 antibodies that have entered the clinic, urelumab and utomilumab, offer a striking example of how differences in epitope, $\mathrm{Fc} \gamma \mathrm{R}$ interactions, ligand-blocking activity, and antibody affinity can influence the activity of TNFRSF agonists $(14,15)$. By optimizing these attributes, we have generated a potentially novel CD137 agonist with an improved efficacy/safety profile.

CTX-471 is a fully human, hinge-stabilized IgG4 antibody that binds to a unique epitope within $\mathrm{CD} 137$ and depends on $\mathrm{Fc} / \mathrm{Fc} \gamma \mathrm{R}$ interactions to deliver a well-tuned agonistic signal. To generate data in mice that would most closely recapitulate pharmacokinetics and pharmacodynamics in humans and cynomolgus monkeys, we additionally generated an affinity matured version (CTX-471-AF) that binds mouse CD137 with comparable affinity as CTX-471 binds to the human receptor. As monotherapies, both CTX-471 and CTX-471-AF exhibited extremely potent antitumor activity in multiple syngeneic mouse models, achieving high rates of complete cures at very low-dose levels. In total, $100 \%$ of mice that rejected their original tumors were fully protected from tumor rechallenge, indicating that CTX-471 promoted long-term immune memory, consistent with the known role of CD137 in expanding memory $\mathrm{T}$ cells in vivo. CTX-471-AF also demonstrated a unique ability to cure large, established CT-26 tumors (450-500 $\mathrm{mm}^{3}$ before treatment), while validated antibodies against other immuno-oncology targets including PD-1, PD-L1, CTLA-4, or OX-40 failed to slow tumor growth. Importantly, the urelumab-like, mouse-specific CD137 agonist antibody $3 \mathrm{H} 3$ was also ineffective in the large tumor model, highlighting the improved in vivo efficacy of CTX-471-AF despite lower in vitro potency.

Immune cell depletion experiments showed that the efficacy of CTX-471 required the presence of $\mathrm{CD}^{+} \mathrm{T}$ cells, $\mathrm{CD}^{+} \mathrm{T}$ cells, and NK cells, indicating a coordinated involvement of both innate and adaptive immune cells. As reported for other TNFR agonist antibodies (20-24), activity of CTX-471 both in vitro and in vivo requires the presence of Fc $\gamma \mathrm{R}$-expressing accessory cells to promote receptor clustering. The selection of a human IgG4 backbone enables coengagement of CD64 (Fc $\gamma$ RI) and CD32b (Fc $\gamma$ RIIb) 
to drive CD137 cross-linking, while limiting the risk of deleterious depletion of CD137 ${ }^{+}$immune effector cells through binding CD16a (Fc $\gamma$ RIIIa). In aggregate, our mechanistic observations indicate that the curative activity of CTX-471 requires not only the presence of CD137-expressing innate and adaptive immune effectors, but also the presence of Fc $\gamma$ R-expressing cells. This codependency provides a good explanation for the tumor-selective activity of CTX-471, as the frequency of both CD137+ TILs and macrophages that constitutively express Fc $\gamma$ Rs have been shown to be higher within tumors as compared with normal tissues (41-44).

At baseline, a high proportion of T cells within CT-26 tumors showed coexpression of the immunoinhibitory receptors PD-1 and TIGIT, a characteristic associated with impaired effector function (45-47). After treatment with CTX-471 or CTX-471-AF, the frequency of intratumoral PD- $1^{+} \mathrm{TIGIT}^{+} \mathrm{T}$ cells decreased significantly, with a more modest effect from $3 \mathrm{H} 3$. In the spleen and liver, CTX-471-AF modestly increased the frequency of $\mathrm{PD}-1^{+} \mathrm{TIGIT}^{+} \mathrm{T}$ cells, while $3 \mathrm{H} 3$ treatment strongly increased peripheral $\mathrm{T}$ cell activation and exhaustion. Similarly, our single-cell gene expression analysis of isolated CD8 $8^{+}$TILs showed that $3 \mathrm{H} 3$ treatment drove a significantly higher proportion of these cells toward exhaustion/dysfunction than did treatment with CTX-471-AF. Together, these observations indicate an altered effector T cell differentiation trajectory elicited by CTX-471, as strong agonists like $3 \mathrm{H} 3$ potentially overstimulate effector $\mathrm{CD}^{+} \mathrm{T}$ cells and promote terminal effector differentiation and exhaustion/dysfunction, whereas the welltuned agonism delivered by CTX-471 appears to sustain a less exhausted/dysfunctional effector phenotype.

By virtue of its unique, nonligand competitive epitope, CTX-471 may synergize with natural CD137/ CD137L signaling. By engaging CD137 with antibody and ligand at the same time, it may be possible to generate larger clusters of the receptor, thereby driving enhanced signaling as reported for other TNFR antibodies (27). Additionally, CD137L expressed on myeloid cells can back signal following CD137 engagement, leading to DC maturation and migration, expression of proinflammatory cytokines and costimulatory receptors, and promotion of a Th1 T cell response in human cellular systems (48), although the effects are less clear in mice (49). Lastly, CD137L back signaling in T cells can limit $\mathrm{T}$ cell activation, an effect that is reversed by CD137 on T cells downregulating the ligand in cis (50). Given these different effects, it is possible that CD137L back signaling may act to tune the primary immune response by promoting myeloid and $\mathrm{T}$ cell activation in a proinflammatory environment but restricting $\mathrm{T}$ cell activation in a low-antigen environment (51). A nonligand competitive antibody such as CTX-471 should retain these natural interactions and regulatory mechanisms, which may be disrupted with a ligand-competitive antibody like utomilumab.

As previously discussed, urelumab showed significant hepatic toxicity in early clinical studies, and similar hepatotoxicity has been recapitulated in mice with $3 \mathrm{H} 3$ (18-20). Other strong agonist antibodies against mouse CD137, including the ligand-blocking clone 2A and nonblocking clone 1D8, also induce liver inflammation, while weaker and FcyR-dependent clone LOB12.3 has no observed hepatotoxicity in mice $(20,52)$. Mechanistic studies using $3 \mathrm{H} 3$ have implicated CD137-mediated activation of liver-resident macrophages, known as Kupffer cells, as the inciting event that leads to a $\mathrm{CD} 8^{+} \mathrm{T}$ cell-driven autoimmune hepatitis (18). In our studies, repeated high doses of CTX-471 or CTX-471-AF did not cause significant changes in hepatic inflammation parameters, whereas $3 \mathrm{H} 3$ triggered liver inflammation with induction of plasma cytokines (TNF- $\alpha$, IFN- $\gamma$, IL-27) and the hepatic stress markers ALT and AST. This differential effect may be explained by macrophages having a higher threshold for CD137-mediated activation than T cells. Additionally, the liver microenvironment may lack features required for CTX-471 to exert its full agonistic effect, such as sufficient density of Fc $\gamma$ R-expressing cells.

Limitations of our studies include potential differences in the structure and function of CD137 in mice and humans, as well as differences in the expression and function of murine and human Fc $\gamma$ Rs. While human IgG4 binds both human and mouse Fc $\gamma \mathrm{RI}$ and Fc $\gamma \mathrm{RIIb}$, there are differences in absolute binding affinity and the extent of effector function across species $(25,34)$.

In conclusion, CTX-471 and CTX-471-AF exhibited extremely potent antitumor activity as monotherapy, resulting in durable cures with generation of protective immune memory. The unique ability of CTX471-AF to completely regress large tumors may represent an unprecedented level of preclinical efficacy for a single-agent immunotherapy. The ability of CTX-471/CTX-471-AF to selectively and profoundly reprogram the tumor microenvironment while sparing unwanted systemic effects indicates that it is an optimally tuned CD137 agonist owing to its unique epitope, requirement for Fc $\gamma \mathrm{R}$ coengagement, and optimized affinity. These attributes of CTX-471 may form a paradigm for the design of optimized agonists targeting other TNFRSF members. Based on our encouraging preclinical findings, CTX-471 has entered clinical development and is currently in a phase I clinical study in patients with solid tumors. 


\section{Methods}

\section{Protein expression and purification}

Antibodies used in the study were generated by cloning DNA encoding both the light and heavy chain sequences independently into the multiple cloning site of the mammalian expression vector pcDNA3.4 (Thermo Fisher Scientific). Sequences for control antibodies, including urelumab and utomilumab, were obtained from the TABS Therapeutic Antibody Database (https://tabs.craic.com) or from PDB files 6MHR and $6 \mathrm{~A} 3 \mathrm{~W}$. Human IgG4 isotype antibodies were generated by fusion of DNA encoding the desired light variable region to the human $\kappa$ constant region (Uniprot P01834) and the desired heavy variable region to the human IgG4 constant region (Uniprot P01861) modified with the S228P mutation to prevent chain shuffling (26). The aglyco-IgG4 variant was generated by mutating the asparagine equivalent to asparagine 297 in human IgG1 (35) to alanine, using standard molecular biology methods. The rat IgG2a isotype antibody was generated by replacing the human constant domains with sequences for rat IgG2a heavy chain and rat $\mathrm{\kappa}$ light chain (Uniprot P20760 and P01836). Subsequently, plasmid DNA for the corresponding light and heavy chains were cotransfected into Expi293 cells (Thermo Fisher Scientific) and incubated for 5 days, and the culture supernatant was harvested. Antibodies were isolated from the resultant supernatants by affinity capture using MabSelect SuRe (GE Healthcare, catalog 11003494), refined by size exclusion chromatography as required and buffer exchanged into PBS, $\mathrm{pH}$ 6.5.

For human CD137 domain mapping, full-length CD137 ECD (Uniprot Q07011; aa 24-186), CRD12 (aa 24-86), or CRD3-4 (aa 87-186) were cloned as fusions with mouse IgG2a-Fc (Uniprot P01863). For Fab fragment affinity measurements, human CD137 ECD (aa 24-186), cynomolgus monkey CD137 ECD (Uniprot A99YE7; aa 24-186), or mouse CD137 ECD (Uniprot P20334; aa 24-187) were cloned as C-terminal His-tag fusions. For human CD137L, the full-length CD137 ligand ECD (Uniprot P41273; aa 50-254) was cloned as a His-tagged fusion. For mapping the contact residues of antibodies with mouse CD137, WT domains 1-4 of CD137 ECD (aa 24-159), CRD3-4 (aa 86-159), and the CRD1-4 mutants (Y40A, Y40R, and K113A) were cloned as fusions with mouse IgG2a-Fc. Protein for recombinant receptor and ligand domains was expressed using Expi293 cells as described for antibody generation above. Depending on the final requirement of these reagents, culture supernatants were either purified by affinity resin or loaded directly onto biosensors to enable binding characterization. For purification, either Ni-NTA Superflow (Qiagen, catalog 30430) or MabSelect SuRe resin were used to isolate His-tagged or Fc fusions, respectively. Subsequently, affinity captured reagents were refined by size exclusion chromatography as required and buffer exchanged into $\mathrm{PBS}, \mathrm{pH} 6.5$.

\section{Antibody binding}

Bio-Layer Interferometry (BLI) binding experiments were performed on Octet HTX (ForteBio) in $1 \times$ PBS, pH 7.4; $0.1 \mathrm{mg} / \mathrm{mL}$ BSA; and $0.002 \%$ Tween 20 . To measure monovalent binding kinetics, histagged CD137 (human, mouse, or cyno) was loaded on prehydrated Ni-NTA biosensors (ForteBio, catalog 18-5102) for 5 minutes. The loaded sensors were incubated with monovalent Fab fragments of CTX-471 or CTX-471-AF at a range of concentrations from 5-500 nM for 5 minutes of association, followed by 15 minutes of dissociation. Results were analyzed with ForteBio Data Analysis 9.0 and fit globally to a 1:1 binding model to determine the monovalent $K_{D}$. For CD137 domain mapping, fulllength CD137 ECD (aa 24-186), CRD 1-2 (aa 24-86), or CRD 3-4 (aa 87-186) expressed as fusions with mouse IgG2a-Fc were captured on anti-mouse-Fc Octet tips (ForteBio, catalog 18-5089). Loaded sensors were incubated with $100 \mathrm{nM}$ of each antibody, and binding response was measured. For ligand competition experiments, antibodies were loaded onto anti-human-Fc tips (ForteBio, catalog 18-5063) and then incubated with $100 \mathrm{nM}$ human CD137, followed by $100 \mathrm{nM}$ human CD137 ligand.

\section{Epitope mapping}

For epitope mapping, a scanning saturation library of CD137 mutants was synthesized with single-point mutations at all noncysteine residue positions to every possible amino acid substitution except cysteine (Twist Biosciences). The library of CD137 variants was displayed on the surface of HEK cells with a single variant per cell and simultaneously stained for binding of nonoverlapping antibodies CTX-471 and urelumab directly conjugated with Alexa Fluor 488 and Alexa Fluor 647 (Thermo Fisher Scientific), respectively. Populations of cells with reduced binding to one antibody but not the other were selected 
on a BD FACSAria Fusion. DNA from sorted populations were analyzed by NGS using an Illumina MiSeq $2 \times 300$ bp platform to identify mutations enriched in each population. Selected CD137 mutants were cloned into His-tagged expression vectors and produced solubly in HEK cells. His-tagged variants were captured directly from the supernatant onto Ni-NTA Octet tips and binding measured to $100 \mathrm{nM}$ CTX-471, urelumab, or CD137-L on Octet HTX (ForteBio). Identified mutations were highlighted on published CD137/CD137L cocrystal structures for human (6CPR) and mouse (6MKZ) using Discovery Studio Visualizer (BioVia).

\section{T cell isolation and preparation}

Human peripheral blood mononuclear cells (PBMCs) were isolated from leukopaks (Stemcell Technologies, catalog 70500) using standard PBMC isolation techniques and frozen down as aliquots in Cryostor-CS10 (Stemcell Technologies, catalog 07930) until the day before an experiment. One day before an assay, PBMCs were thawed and rested overnight in T cell media (TCM). For functional assays, total $\mathrm{T}$ cells were isolated using an EasySep Human T Cell Isolation Kit (Stemcell Technologies, catalog 17951). For mouse $\mathrm{T}$ cell assays, BALB/c mouse (Charles River Laboratories, strain BALB/cAnNCrl) spleens were perfused with Sorting Buffer (PBS + 1\% FBS +2 mM EDTA) to isolate splenocytes. The isolated splenocytes were passed through a 70- $\mu \mathrm{m}$ nylon mesh filter and then directly used for CD ${ }^{+} \mathrm{T}$ cell isolation (Miltenyi Biotec, catalog 130-104-075) without RBC lysis. For Cynomolgus macaque $\mathrm{T}$ cell assays, cynomolgus PBMCs (IQ Biosciences, catalog IQB-MnPB102) were thawed, resuspended in TCM at $2 \times 10^{6} / \mathrm{mL}$, and then activated by adding anti-CD3 (Mabtech, catalog 3610-1-50) at $1 \mu \mathrm{g} /$ $\mathrm{mL}$ for 3 days. After 3 days, the expanding cells were cultured in TCM containing $5 \mathrm{ng} / \mathrm{mL}$ hIL-2 (BioLegend, catalog 589106) and $2.5 \mathrm{ng} / \mathrm{mL}$ IL-7 (BioLegend, catalog 581904) for an additional 8-12 days, with media changes every 2-3 days, before being switched back to cytokine-free TCM the day before setting up an assay.

\section{T cell activation assays}

For in-culture functional studies with primary human T cells, 100,000 total T cells were cocultured with 50,000 ExpiCHO-S cells (CHO, Thermo Fisher Scientific, catalog A29127) lentivirally transduced to express human CD32b (CHO-CD32b). Anti-CD137 or isotype control antibodies were added at indicated concentration, along with $0.25 \mu \mathrm{g} / \mathrm{mL}$ anti-human CD3 antibody (clone OKT-3, Thermo Fisher Scientific, catalog 16-0037-8). After a 3-day coculture, supernatants were tested for secreted IFN- $\gamma$ using an electrochemiluminescence assay (Meso Scale Discovery, catalog K151AEB-4). In experiments testing the effect of CD137L on antibody activity, $0.5 \mu \mathrm{g} / \mathrm{mL}$ CD137-Fc was added to each well.

For in-culture functional studies with primary mouse and cynomolgus monkey $\mathrm{T}$ cells, a similar procedure was employed with the following deviations. Mouse T cells were cocultured with K562CD32 cells instead of CHO-CD32 due to an observed ability of $\mathrm{CHO}$ cells to provide endogenous costimulation to mouse $\mathrm{T}$ cells. The anti-mouse CD3 antibody (clone 145-2C11, BioLegend, catalog 100331) and the mouse IFN- $\gamma$ detection kit (Meso Scale Diagnostics, catalog K152AEB-2) were used. Cynomolgus $\mathrm{T}$ cells were activated in the cocultures using anti-monkey CD3 antibody (clone CD3-1, mAbTech, catalog 3610-1-50) and IFN- $\gamma$ measured with a nonhuman primate detection kit (Meso Scale Diagnostics, catalog K156QOD-1).

\section{Receptor occupancy (RO)}

Primary $\mathrm{CD}^{+} \mathrm{T}$ cells from humans and mice or total $\mathrm{T}$ cells from cynomolgus monkeys were activated with CD3 antibodies as described above. Total $\mathrm{T}$ cells from cynomolgus monkeys were further stained with CD8 (BioLegend, catalog 301032) and CD4 (eBioscience, catalog 12-4998-82) antibodies and gated on $\mathrm{CD}^{+}$prior to analysis. Activated T cells were incubated with serial dilutions of CTX-471 or CTX471-AF (0.003-100 nM) overnight, and binding was detected with a secondary antibody against human IgG (eBioscience, catalog 12-4998-82). Staining with saturating concentrations of CTX-471 or CTX-471AF was used to establish the mean fluorescence intensity (MFI) with $100 \%$ RO (MFI $100 \%$ Ro $)$. Binding MFI of an isotype control antibody was considered background and subtracted from all values. Percent RO was calculated using the background subtracted values as $\% \mathrm{RO}=100 \times\left(\mathrm{MFI}_{\text {Sample }} / \mathrm{MFI}_{100 \% \mathrm{RO}}\right)$. In parallel, a noncompetitive antibody was used to determine total CD137 levels. 


\section{Clustering and signaling assays}

HEK-SplitCD137 cells were generated by transducing HEK-293 cells (American Type Culture Collection [ATCC], catalog ATCC-CRL-1573) with lentiviral constructs expressing human CD137 fused to either amino acids $2-416$ of firefly luciferase with a neomycin selection cassette or amino acids 398-550 of firefly luciferase with a puromycin selection cassette. Cells stably expressing both fusion proteins were selected by culturing in $1 \mu \mathrm{g} / \mathrm{mL}$ puromycin and $100 \mathrm{ng} / \mathrm{mL}$ neomycin for 2 weeks.

For measuring CD137 clustering, CD137 and isotype control antibodies were incubated for 30 minutes with the anti-human $\mathrm{F}\left(\mathrm{Ab}^{\prime}\right)_{2}$ reagent (Jackson ImmunoResearch, catalog 109-006-098) to induce cross-linking. A total of 50,000 HEK-SplitCD137 cells were incubated with serial dilutions of the cross-linked antibodies for 4 hours. The cells were lysed with Bright-Glo substrate (Promega, catalog E2620), and the resulting luminescence signals were measured using the BioTek Synergy $\mathrm{H} 1$ microplate reader.

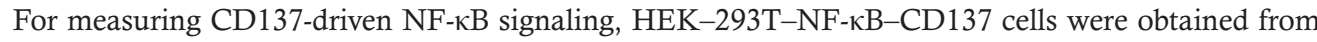
CrownBio (catalog C2012). A subclone designated $1 \mathrm{E} 17$ was selected for optimal signaling properties and used in these assays in the presence or absence of plate-bound $10 \mathrm{nM} \mathrm{CD137L-Fc} \mathrm{(Sino} \mathrm{Biological,} \mathrm{catalog}$

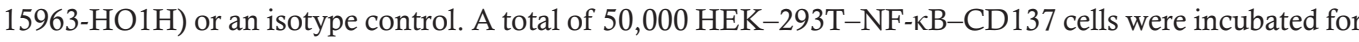
4 hours with serial dilutions of the CD137 or isotype control antibodies. The cells were then lysed by addition of $100 \mu \mathrm{L}$ per well SteadyLite plus substrate buffer (Perkin Elmer, catalog 6066751), and the resulting luminescence signals were measured using the BioTek Synergy H1 microplate reader.

\section{Syngeneic tumor models}

Cell lines, tumor formation, and tumor monitoring. CT-26, A20, and EMT6 cell lines were obtained from ATCC. CT-26 colon carcinoma cells were cultured in DMEM containing 10\% heat-inactivated FBS and maintained at $37^{\circ} \mathrm{C}$ and $5 \% \mathrm{CO}_{2}$. For culturing EMT6 breast carcinoma cells, the medium was further supplemented with $1 \mathrm{mM}$ sodium pyruvate solution, $1 \times$ NEAA solution, and $1 \times$ MEM vitamin solution. A20 B cell lymphoma cells were cultured in RPMI 1640 media, containing 10\% heat-inactivated FBS, $10 \mathrm{mM}$ HEPES, $1 \mathrm{mM}$ sodium pyruvate, and $0.05 \mathrm{mM}$ 2-mercaptoethanol. All cell lines were tested and verified to be murine virus and mycoplasma free prior to in vivo implantation.

BALB/c mice were obtained from Charles River Laboratories and were 6-9 weeks old at the beginning of study. A total of $1 \times 10^{5} \mathrm{CT}-26$ cells or $5 \times 10^{6}$ A20 cells were injected s.c. into the right flank in $0.1 \mathrm{~mL} \mathrm{PBS}$. A total of $5 \times 10^{4}$ EMT6 cells were injected into the right mammary fat pad in $0.05 \mathrm{~mL}$ PBS. Mice were randomized into groups of 6-10, and dosing was initiated 7-14 days after tumor cell inoculation when the tumors reached a predetermined volume $\left(50-100 \mathrm{~mm}^{3}\right.$ for standard tumor models and $450-500 \mathrm{~mm}^{3}$ for large tumor models). Tumor width and length were measured using dial calipers, and tumor volumes were calculated by the formula length $\times\left(\right.$ width $\left.^{2}\right) / 2$. Mice were euthanized when the tumor size reached the humane endpoint (2000 $\mathrm{mm}^{3}$ for CT-26 and A20 tumor-bearing mice; $1000 \mathrm{~mm}^{3}$ for EMT6 tumor-bearing mice). Mice with no palpable evidence of tumors for 60-90 days after termination of treatment were considered cured and were subsequently rechallenged with the same tumor cells of the on the opposite (left) flank. As control, 5 naive mice were also inoculated in the same manner.

Treatments. Antibodies were diluted in sterile citrate buffer (20 mM Citrate, $150 \mathrm{mM} \mathrm{NaCl}, \mathrm{pH}$ 6.0) and i.p. administered. CTX-471, CTX-471-AF, and 3H3 (BioXcell, catalog BE0239) were given at doses ranging from $12.5-200 \mu \mathrm{g} /$ mouse either at days $0,3,6$, and 9 or at days $0,7,14,21$, and 28 as indicated in the figure legends. For the comparative large-tumor experiment, clinical grade Avelumab (anti-PD-L1) was sourced from Myoderm (NDC 44087-3535-01), whereas RMP1-14 (anti-PD-1, catalog BE0146), 9H10 (anti-CTLA-4, catalog BP0131), and OX-86 (anti-OX-40, catalog BE0031) were purchased from BioXcell. Mice with approximately $450 \mathrm{~mm}^{3}$ CT-26 tumors were administered with CD137 agonists (CTX-471-AF or $3 \mathrm{H} 3 ; 25 \mu \mathrm{g}$ /mouse on days $0,7,14$ ), checkpoint inhibitors (Avelumab, RMP1-14, or 9H10; $200 \mu \mathrm{g}$ / mouse on days $0,3,6$ ), or an OX40 agonist (OX-86; $200 \mu \mathrm{g}$ /mouse on days $0,3,6$ ).

In T cell depletion experiments, mice ( $n=10$ per group) were administered $500 \mu \mathrm{g} / \mathrm{mouse}$ of CD4 (GK1.5, BioXcell, catalog BE0003) or CD8 (YTS 169.4, BioXcell, catalog BP0117) antibodies on days -1 , $0,5,10,15$, and 20 after CT-26 tumor cell implantation and treated with $150 \mu \mathrm{g} /$ mouse CTX-471 on days $6,9,12,19$, and 26 . In NK cell-depletion experiments, mice ( $n=8$ per group) were administered $50 \mu \mathrm{L} /$ mouse of asialo-GM1 antibody (anti-ASGM1, BioLegend, catalog 146002) on days -1, 0, 5, 10, 15, and 20 after CT-26 tumor cell implantation and treated with $150 \mu \mathrm{g} /$ mouse CTX-471 on days 7, 10, 13, 20, and 27. Depletion of $\mathrm{T}$ and NK cells in the peripheral blood was confirmed by flow cytometry. 
Immune profiling. Mice were euthanized by $\mathrm{CO}_{2}$ asphyxiation, and tumors, spleen, and livers were harvested at indicated time points after treatment with CTX-471, CTX-471-AF, or 3H3. Tumor tissues were enzymatically digested using tumor dissociation kit components (Miltenyi Biotec, catalog 130-096-730) and mechanically disrupted with a gentleMACS dissociator (Miltenyi Biotec). Cell suspensions were prepared from spleens by mechanical disruption, followed by lysis of RBC (MilliporeSigma, catalog R7757). Liver tissues were enzymatically digested using liver dissociation kit components (Miltenyi Biotec, catalog 130-105-807) combined with gentleMACS disruption. Following tissue digestion, debris was separated by sedimentation in Lympholyte-M cell separation media (Thermo Fisher Scientific, catalog CL5035). Resulting suspensions from tumors, spleens, and livers were passed through a $40-\mu \mathrm{m}$ nylon cell strainer.

Single cell suspensions were stained with fixable viability dye (eBioscience, catalog 65-0865-18) and Fc receptors blocked with anti-CD16/CD32 (eBioscience, catalog 13-0161-86). For cell surface staining, single cell suspensions were incubated with antibody cocktails for 30 minutes on ice. Intercellular staining was performed using the FoxP3/transcription factor staining buffer set following the manufacturer's fixation/ permeabilization protocol (eBioscience, catalog 00-5523-00). The optimal concentration for each antibody was predetermined by titration.

Fluorophore-conjugated antibodies against CD45 (clone 30-F11), CD11b (clone M1/70), FOXP3 (clone FJK-16s), F4/80 (clone BM8), CD44 (clone IM7), TIGIT (clone GIGD7), and iNOS (clone CXNFT) were obtained from eBioscience. Antibodies against CD4 (clone RM4-5), CD8 (clone 53-6.7), CD25 (clone PC61.5), CD137 (clone 1AH2), and CD38 (clone Ab90) were purchased from BD Biosciences. CD3 (clone 17A2), PD-1 (clone RMP1-14), and CD62L (clone MEL-14) antibodies were purchased from BioLegend. MACSQuant cytometer (Miltenyi Biotec) or LSR Fortesa (BD Biosciences) were used to analyze stained cells. Fluorescence minus one control were used for each marker to determine the placement of positive gates. Data analysis was performed using FlowJo software (version 10).

\section{Differential gene expression analysis of CD8 ${ }^{+}$TILs}

$\mathrm{CD}^{+} \mathrm{T}$ cells were sorted from CT-26 tumor-bearing mice that were treated with CTX-471-AF, 3H3, or isotype control antibodies and were encapsulated into droplets. Libraries were prepared using Chromium Single Cell 3' Reagent Kits v2 according to the manufacturer's protocol (10× Genomics). The generated scRNA-Seq libraries were sequenced using a 75-cycle Nextseq 500 high-output V2 kit. Read demultiplexing, mm10 reference alignment, filtering, Unique Molecular Identifier-collapsing (UMI-collapsing), and expression matrix generation was performed using Cellranger 2.1.0 (10× Genomics). Cells with fewer than 400 genes detected or genes expressed in fewer than 3 cells were removed from analysis. UMI counts were normalized to account for differences in coverage such that each row (cell) in the expression matrix adds to 10,000 . Data was then log-transformed $\log _{2}(T P M+1)$ and scaled to a mean of 0 with unit variance for further analysis using Scanpy (Version 1.4). Data were deposited in the Gene Expression Omnibus (https://www.ncbi.nlm.nih.gov/geo/) with accession number GSE144473.

For data visualization, t-distributed stochastic neighbor embedding (t-SNE), as implemented in Scikit-learn (http://www.jmlr.org/papers/v12/pedregosa11a.html), was applied with a perplexity of 30 calculated from the first 20 principal components as evaluated on highly variable genes. To cluster cells, the Louvain graph clustering method was implemented by https://zenodo.org/record/35117 with resolution parameter 0.6 computed on a nearest neighbors graph with $\mathrm{k}=40$ nearest neighbors. Two clusters enriched for DC and $\mathrm{B}$ cell signatures were removed and reclustered on the nearest neighbors graph with $\mathrm{k}=40$ neighbors and perplexity of 0.6 again.

For each signature (a set of genes), each cell was scored by its average expression across the signature list, subtracted from 50 randomly subsampled genes with similar expression as defined by 25 gene expression bins using the "score_genes" function as implemented in Scanpy. For signatures with both upregulated and downregulated genes, scores were calculated and separated, and the final score was down-signature subtracted from the up-signature. A 1-way ANOVA test of expression between samples was applied to evaluate statistical shifts in signature enrichment. In all signature comparisons, $P$ values from the F-distribution were highly significant. Additionally, Tukey's pairwise range test was used to evaluate differences of enrichment between samples. To control for FDR, the Benjamini-Hochberg procedure was used as implemented by the Python stats models package with $\alpha=0.05$. To calculate the significance of gene expression between groups of cells, Benjamini-Hochberg-corrected $P$ values from a 2-tailed $t$ test with intentionally overestimated variance were used to reduce the likelihood of false positives as implemented in Scanpy's "rank_genes_groups" function. 


\section{Assessment of hepatic inflammation}

Potential for CD137 agonist-induced hepatic inflammation was assessed in a dose-ranging safety pharmacology study in nontumor bearing C57BL/6 mice. Mice received a citrate buffer negative control, CTX471, CTX-471-AF, or antibody $3 \mathrm{H} 3$ by weekly i.v. bolus injections on days $0,7,14$, and 21 . CTX-471 and CTX-471-AF were tested at 10, 20, 40, and $80 \mathrm{mg} / \mathrm{kg}$ dose levels, and antibody $3 \mathrm{H} 3$ was tested at 10 and $80 \mathrm{mg} / \mathrm{kg}$. Livers and spleens were harvested and weighed on day 28. Intrahepatic and splenic CD $8^{+}$ $\mathrm{T}$ cell levels were measured by flow cytometry as described above. Plasma levels of liver transaminase enzymes were measure using ALT activity assay (MilliporeSigma, catalog MAK052) and AST activity assay (MilliporeSigma, catalog MAK055) kits. Plasma levels of proinflammatory cytokines were measured with custom multiplexed electrochemiluminescence assay (Meso Scale Discovery) in accordance with the manufacturer's protocol for evaluation of mouse cytokine levels in plasma.

For histological analysis, liver lobes were collected in histology cassettes and submerged into $10 \%$ neutral buffered formalin for 24 hours prior to transferring into $70 \%$ ethanol for long-term storage. Tissues were than embedded in paraffin, sectioned, and stained with H\&E. Immunohistochemical staining with anti-mouse CD8 (Cell Signaling Technologies, catalog 98941) and anti-mouse F4/80 (Cell Signaling Technologies, catalog 70076) antibodies were performed on serial sections using standard methods. Numbers of $\mathrm{CD}^{+}$cells, $\mathrm{F} 4 / 80^{+}$cells, or $\mathrm{F} 4 / 80^{+}$clusters (defined as $>10$ cells) were counted using the QuPath software (version 0.1.2).

\section{Macrophage differentiation and activation}

For differentiation of macrophages, unfractionated mouse BM (femurs and tibias) or human $\mathrm{CD} 14^{+}$ peripheral blood monocytes were utilized. $\mathrm{CD} 14^{+}$monocytes were isolated using ferromagnetic bead separation (Miltenyi Biotec). Cells were matured in vitro to macrophages using either mouse or human M-CSF (Shenandoah Biotechnology) at $20 \mathrm{ng} / \mathrm{mL}$ for a period of 7 days. At the end of maturation culture, adherent macrophages were harvested and reseeded in 96-well plates and rested overnight in minimal media without additional cytokines. Cells were then reactivated with $10 \mu \mathrm{g} / \mathrm{mL}$ CpG ODN (multispecies specific D-SL01, Invivogen) and the indicated antibodies for a period of 2 days. Following reactivation, cytokine production in the supernatant was analyzed using a multiplexed electrochemiluminescence-linked immunosorbent assay (Meso Scale Discovery).

Data and materials availability

Requests for materials should be addressed to TS and will be provided pending completion of a material transfer agreement with Compass Therapeutics

\section{Statistics}

For in vivo studies, the statistical significance of differences between treatment groups was calculated using GraphPad Prism (version 7). Log-rank test followed by Bonferroni's multiple comparisons test was used to determine statistical significance between Kaplan-Meier survival curves. For all other data, 1-way ANOVA with Bonferroni's multiple comparisons test was performed. Significance were indicated as follows: ${ }^{*} P<0.05 ;{ }^{* *} P<0.01 ;{ }^{* *} P<0.001 ;{ }^{* * *} P<0.0001$. All data are represented as mean \pm SEM.

\section{Study approval}

All animal studies were performed according to the guidelines of the IACUC at Compass Therapeutics.

\section{Author contributions}

UE designed in vivo pharmacology studies, oversaw the execution of all in vivo experiments, and interpreted the data. WG performed in vivo experiments and immunophenotyping studies. BW designed and executed in vitro functional assays and interpreted the data. CC performed in vivo studies. LM performed in vivo studies. HJW performed in vivo studies. MO designed and performed in vitro signaling studies. CL analyzed scRNA-Seq data. CW oversaw analysis of scRNA-Seq data. PB performed in vitro functional assays. DCG performed in vitro cell binding assays. SO performed biochemical binding studies. LL designed and oversaw biochemical binding studies. WKM expressed and purified truncated variants of CD137. SQH cloned and prepared DNA encoding truncated variants of CD137. CLL designed and executed epitope mapping studies. JL designed and executed affinity maturation experiments and 
performed structural analyses. WFC designed and performed in vitro macrophage assays. NZ led the early functional characterization efforts. ACA supervised the scRNA-Seq analysis methods and interpreted the data. MMS led the antibody discovery and engineering research efforts. PB provided overall scientific critique and guidance. TJS provided overall scientific critique and guidance. RT led the pharmacology research efforts, provided overall guidance, and interpreted the data. All authors contributed to the drafting and critical revision of the manuscript and approved the final version of the manuscript.

\section{Acknowledgments}

We thank the research teams at Compass Therapeutics. We thank Autumn Ruiz, Puru Nanjappa, Sujan Lama, Beata Bobrowicz, Pia Muyot, and Alan Nhan for their technical contributions. This study was supported by Compass Therapeutics LLC.

Address correspondence to: Tom Schuetz, Compass Therapeutics, 245 First Street, Cambridge, 02142, USA. Phone: 617.500.8099; Email: thomas.schuetz@compasstherapeutics.com.

1. Mayes PA, Hance KW, Hoos A. The promise and challenges of immune agonist antibody development in cancer. Nat Rev Drug Discov. 2018;17(7):509-527.

2. Makkouk A, Chester C, Kohrt HE. Rationale for anti-CD137 cancer immunotherapy. Eur J Cancer. 2016;54:112-119.

3. Fesnak AD, June CH, Levine BL. Engineered T cells: the promise and challenges of cancer immunotherapy. Nat Rev Cancer. 2016;16(9):566-581.

4. Li G, et al. 4-1BB enhancement of CAR T function requires NF-אB and TRAFs. JCI Insight. 2018;3(18):121322.

5. Melero I, et al. Monoclonal antibodies against the 4-1BB T-cell activation molecule eradicate established tumors. Nat Med. 1997;3(6):682-685.

6. Vinay DS, Kwon BS. Immunotherapy of cancer with 4-1BB. Mol Cancer Ther. 2012;11(5):1062-1070.

7. Houot R, et al. Therapeutic effect of CD137 immunomodulation in lymphoma and its enhancement by Treg depletion. Blood. 2009;114(16):3431-3438.

8. Bartkowiak T, Curran MA. 4-1BB Agonists: Multi-Potent Potentiators of Tumor Immunity. Front Oncol. 2015;5:117.

9. Ochoa MC, et al. Daratumumab in combination with urelumab to potentiate anti-myeloma activity in lymphocyte-deficient mice reconstituted with human NK cells. Oncoimmunology. 2019;8(7):1599636.

10. Masu T, et al. Anti-CD137 monoclonal antibody enhances trastuzumab-induced, natural killer cell-mediated cytotoxicity against pancreatic cancer cell lines with low human epidermal growth factor-like receptor 2 expression. PLoS One. 2018;13(12):e0200664.

11. Srivastava RM, et al. CD137 Stimulation Enhances Cetuximab-Induced Natural Killer: Dendritic Cell Priming of Antitumor T-Cell Immunity in Patients with Head and Neck Cancer. Clin Cancer Res. 2017;23(3):707-716.

12. Chen S, et al. Combination of 4-1BB agonist and PD-1 antagonist promotes antitumor effector/memory CD8 T cells in a poorly immunogenic tumor model. Cancer Immunol Res. 2015;3(2):149-160.

13. Uno T, et al. Eradication of established tumors in mice by a combination antibody-based therapy. Nat Med. 2006;12(6):693-698.

14. Segal NH, et al. Results from an Integrated Safety Analysis of Urelumab, an Agonist Anti-CD137 Monoclonal Antibody. Clin Cancer Res. 2017;23(8):1929-1936.

15. Segal NH, et al. Phase I Study of Single-Agent Utomilumab (PF-05082566), a 4-1BB/CD137 Agonist, in Patients with Advanced Cancer. Clin Cancer Res. 2018;24(8):1816-1823.

16. Chin SM, et al. Structure of the 4-1BB/4-1BBL complex and distinct binding and functional properties of utomilumab and urelumab. Nat Commun. 2018;9(1):4679.

17. Shuford WW, et al. 4-1BB costimulatory signals preferentially induce CD8+ T cell proliferation and lead to the amplification in vivo of cytotoxic T cell responses. J Exp Med. 1997;186(1):47-55.

18. Bartkowiak T, et al. Activation of 4-1BB on Liver Myeloid Cells Triggers Hepatitis via an Interleukin-27-Dependent Pathway. Clin Cancer Res. 2018;24(5):1138-1151.

19. Niu L, et al. Cytokine-mediated disruption of lymphocyte trafficking, hemopoiesis, and induction of lymphopenia, anemia, and thrombocytopenia in anti-CD137-treated mice. J Immunol. 2007;178(7):4194-4213.

20. Qi X, et al. Optimization of 4-1BB antibody for cancer immunotherapy by balancing agonistic strength with Fc $\gamma$ R affinity. Nat Commun. 2019;10(1):2141.

21. Yu X, et al. Complex Interplay between Epitope Specificity and Isotype Dictates the Biological Activity of Anti-human CD40 Antibodies. Cancer Cell. 2018;33(4):664-675.e4.

22. Dahan R, Barnhart BC, Li F, Yamniuk AP, Korman AJ, Ravetch JV. Therapeutic Activity of Agonistic, Human Anti-CD40 Monoclonal Antibodies Requires Selective Fc $\gamma$ R Engagement. Cancer Cell. 2016;29(6):820-831.

23. Buchan SL, et al. Antibodies to Costimulatory Receptor 4-1BB Enhance Anti-tumor Immunity via T Regulatory Cell Depletion and Promotion of CD8 T Cell Effector Function. Immunity. 2018;49(5):958-970.e7.

24. Zhang D, Goldberg MV, Chiu ML. Fc Engineering Approaches to Enhance the Agonism and Effector Functions of an AntiOX40 Antibody. J Biol Chem. 2016;291(53):27134-27146.

25. Derebe MG, Nanjunda RK, Gilliland GL, Lacy ER, Chiu ML. Human IgG subclass cross-species reactivity to mouse and cynomolgus monkey Fcy receptors. Immunol Lett. 2018;197:1-8.

26. Davies AM, Sutton BJ. Human IgG4: a structural perspective. Immunol Rev. 2015;268(1):139-159.

27. Vanamee ÉS, Faustman DL. Structural principles of tumor necrosis factor superfamily signaling. Sci Signal. 
2018;11(511):eaao4910.

28. Jang IK, Lee ZH, Kim YJ, Kim SH, Kwon BS. Human 4-1BB (CD137) signals are mediated by TRAF2 and activate nuclear factor-kappa B. Biochem Biophys Res Commun. 1998;242(3):613-620.

29. Teijeira A, et al. Mitochondrial Morphological and Functional Reprogramming Following CD137 (4-1BB) Costimulation. Cancer Immunol Res. 2018;6(7):798-811.

30. Menk AV, et al. 4-1BB costimulation induces T cell mitochondrial function and biogenesis enabling cancer immunotherapeutic responses. J Exp Med. 2018;215(4):1091-1100.

31. Kurtulus S, et al. Checkpoint Blockade Immunotherapy Induces Dynamic Changes in PD-1-CD ${ }^{+}$Tumor-Infiltrating T Cells. Immunity. 2019;50(1):181-194.e6.

32. Singer M, et al. A Distinct Gene Module for Dysfunction Uncoupled from Activation in Tumor-Infiltrating T Cells. Cell. 2016;166(6):1500-1511.e9.

33. Dekkers G, et al. Affinity of human IgG subclasses to mouse Fc gamma receptors. MAbs. 2017;9(5):767-773

34. Overdijk MB, et al. Crosstalk between human IgG isotypes and murine effector cells. J Immunol. 2012;189(7):3430-3438

35. Ju MS, Jung ST. Aglycosylated full-length IgG antibodies: steps toward next-generation immunotherapeutics. Curr Opin Biotechnol. 2014;30:128-139.

36. Dahan R, Sega E, Engelhardt J, Selby M, Korman AJ, Ravetch JV. Fc $\gamma$ Rs Modulate the Anti-tumor Activity of Antibodies Targeting the PD-1/PD-L1 Axis. Cancer Cell. 2015;28(3):285-295.

37. Arce Vargas F, et al. Fc-Optimized Anti-CD25 Depletes Tumor-Infiltrating Regulatory T Cells and Synergizes with PD-1 Blockade to Eradicate Established Tumors. Immunity. 2017;46(4):577-586.

38. Lau J, et al. Tumour and host cell PD-L1 is required to mediate suppression of anti-tumour immunity in mice. Nat Commun 2017;8:14572.

39. Selby MJ, et al. Anti-CTLA-4 antibodies of IgG2a isotype enhance antitumor activity through reduction of intratumoral regulatory T cells. Cancer Immunol Res. 2013;1(1):32-42.

40. Yu JW, et al. Tumor-immune profiling of murine syngeneic tumor models as a framework to guide mechanistic studies and predict therapy response in distinct tumor microenvironments. PLoS One. 2018;13(11):e0206223

41. Ye Q, et al. CD137 accurately identifies and enriches for naturally occurring tumor-reactive T cells in tumor. Clin Cancer Res. 2014;20(1):44-55.

42. Jung KY, et al. Cancers with Higher Density of Tumor-Associated Macrophages Were Associated with Poor Survival Rates. J Pathol Transl Med. 2015;49(4):318-324.

43. Yang J, Li X, Liu X, Liu Y. The role of tumor-associated macrophages in breast carcinoma invasion and metastasis. Int J Clin Exp Pathol. 2015;8(6):6656-6664.

44. Singhal S, et al. Human tumor-associated monocytes/macrophages and their regulation of T cell responses in early-stage lung cancer. Sci Transl Med. 2019;11(479):eaat1500.

45. Dixon KO, et al. Functional Anti-TIGIT Antibodies Regulate Development of Autoimmunity and Antitumor Immunity. J Immunol. 2018;200(8):3000-3007.

46. Chauvin JM, et al. TIGIT and PD-1 impair tumor antigen-specific CD8+ T cells in melanoma patients. J Clin Invest. 2015;125(5):2046-2058.

47. Hung AL, et al. TIGIT and PD-1 dual checkpoint blockade enhances antitumor immunity and survival in GBM. Oncoimmunology. 2018;7(8):e1466769.

48. Zeng Q, Zhou Y, Schwarz H. CD137L-DCs, Potent Immune-Stimulators-History, Characteristics, and Perspectives. Front Immunol. 2019;10:2216

49. Tang Q, Jiang D, Shao Z, Martínez Gómez JM, Schwarz H. Species difference of CD137 ligand signaling in human and murine monocytes. PLoS One. 2011;6(1):e16129.

50. Eun SY, Lee SW, Xu Y, Croft M. 4-1BB ligand signaling to T cells limits T cell activation. J Immunol. 2015;194(1):134-141.

51. Kwon B. Is CD137 Ligand (CD137L) Signaling a Fine Tuner of Immune Responses? Immune Netw. 2015;15(3):121-124

52. Dubrot J, et al. Treatment with anti-CD137 mAbs causes intense accumulations of liver T cells without selective antitumor immunotherapeutic effects in this organ. Cancer Immunol Immunother. 2010;59(8):1223-1233. 